\title{
CATEGORICAL ASPECTS OF TORIC TOPOLOGY
}

\author{
TARAS E PANOV AND NIGEL RAY
}

\begin{abstract}
We argue for the addition of category theory to the toolkit of toric topology, by surveying recent examples and applications. Our case is made in terms of toric spaces $X_{K}$, such as moment-angle complexes $\mathcal{Z}_{K}$, quasitoric manifolds $M$, and Davis-Januszkiewicz spaces $D J(K)$. We first exhibit $X_{K}$ as the homotopy colimit of a diagram of spaces over the small category $\mathrm{CAT}(K)$, whose objects are the faces of a finite simplicial complex $K$ and morphisms their inclusions. Then we study the corresponding $\mathrm{CAT}(K)$-diagrams in various algebraic Quillen model categories, and interpret their homotopy colimits as algebraic models for $X_{K}$. Such models encode many standard algebraic invariants, and their existence is assured by the Quillen structure. We provide several illustrative calculations, often over the rationals, including proofs that quasitoric manifolds (and various generalisations) are rationally formal; that the rational Pontrjagin ring of the loop space $\Omega D J(K)$ is isomorphic to the quadratic dual of the Stanley-Reisner algebra $\mathbb{Q}[K]$ for flag complexes $K$; and that $D J(K)$ is coformal precisely when $K$ is flag. We conclude by describing algebraic models for the loop space $\Omega D J(K)$ for any complex $K$, which mimic our previous description as a homotopy colimit of topological monoids.
\end{abstract}

\section{INTRODUCTION}

1.1. Toric Topology. Toric topology is rapidly gaining recognition as an area of independent mathematical interest, and the aim of this article is to survey aspects of category theory that are exerting a growing influence on its development. The primary objects of study are derived from well-behaved actions of the $n$-dimensional torus $T^{n}$ on a topological space, and lie in a variety of geometric and algebraic categories. We therefore refer to them as toric objects. Each of the orbit spaces is equipped with a natural combinatorial structure, which encodes the distribution of isotropy subgroups and is determined by a finite simplicial complex $K$. In this context, we wish to describe and evaluate topological and homotopy theoretic invariants of toric spaces in terms of combinatorial data associated to $K$.

A fundamental example is provided by the moment-angle complex $\mathcal{Z}_{K}$, 8 , which depends only on the choice of $K$. When $K$ has $m$ vertices, $\mathcal{Z}_{K}$ supports a canonical action of $T^{m}$ whose quotient is naturally homeomorphic to the cone $C K^{\prime}$ on the barycentric subdivision of $K$. If $K$ is the boundary of an $n$-dimensional simplicial polytope then $C K^{\prime}$ may be identified with its dual polytope $P$, which is simple. In this case, certain subtori $T^{m-n}<T^{m}$ act freely on $\mathcal{Z}_{K}$, and their quotient spaces $M^{n}$ are the toric manifolds introduced by Davis and Januszkiewicz [11]. In order to avoid confusion with the toric varieties of algebraic geometry, we follow current

2000 Mathematics Subject Classification. Primary 55U35, 57R91; Secondary 57N65, 55P35.

Key words and phrases. Homotopy colimit, model category, toric topology.

The first author was supported by EPSRC Visiting Fellowship GR/S55828/01 at the University of Manchester, by the Russian Foundation for Basic Research (grants 08-01-00541 and 08-0191855-KO), and by P Deligne's 2004 Balzan Prize for Mathematics. 
convention $[8$ by labelling them quasitoric manifolds. The quotient of any such $M$ by the $n$-torus $T^{m} / T^{m-n}$ is naturally homeomorphic to $P$.

It is convenient to describe several families of toric spaces $X_{K}$ as colimits of diagrams of topological spaces over the small category CAT $(K)$, whose objects are the faces of $K$ and morphisms their inclusions. In certain cases, algebraic invariants of $X_{K}$ may then be described in terms of the corresponding diagrams in appropriate algebraic categories; in other words, by algebraic toric objects. A typical example is given by the Davis-Januszkiewicz space $D J(K)$. As introduced in [11, $D J(K)$ is defined by the Borel construction $\mathcal{Z}_{K} \times_{T^{m}} E T^{m}$, and its cohomology ring $H^{*}(D J(K) ; R)$ is isomorphic to the Stanley-Reisner algebra $R[K]$ [44 for any coefficient ring $R$. Following 8 and 36, it may be interpreted as the colimit of a diagram $B T^{K}$, whose value on each face $\sigma$ of $K$ is the cartesian product $B T^{\sigma}$; then $R[K]$ is isomorphic to the limit of the corresponding $\mathrm{CAT}^{o p}(K)$-diagram of polynomial algebras $S_{R}(K)$, whose value on $\sigma$ is the polynomial algebra $S_{R}(\sigma)$ generated by its vertices.

Such constructions involve the categories $\operatorname{CAT}(K)$ in a local rôle, and are already well-publicised. We believe that category theory also offers an important global viewpoint, which suggests a systematic programme for the evaluation of algebraic and geometrical invariants of $X_{K}$.

1.2. Categorical Motivation. The motivation for our programme lies in two closely related observations about the local situation. One is that many familiar invariants of toric spaces depend only on their homotopy type, yet homotopy equivalences do not interact well with colimits; the other is that many of the functors we wish to apply to $X_{K}$ do not respect colimits, and may therefore be difficult to evaluate. If, however, we can justify interpreting $X_{K}$ as a homotopy colimit [6], such difficulties often evaporate. This possibility seems first to have been recognised by Welker, Ziegler, and Živaljević [48, who show that toric varieties themselves are expressible as homotopy colimits. Our own evidence is provided in [36], where the loop space $\Omega D J(K)$ is modelled geometrically by a homotopy colimit in the category TMON of topological monoids. Developing these themes leads us naturally into the world of Quillen model categories [38.

We therefore work with model categories whenever we are able. As well as being currently fashionable, Quillen's theory suggests questions that we might not otherwise have asked, and presents challenges of independent interest. For example, the existence of homotopy colimits in an arbitrary model category has been known for some time [23, but specific constructions are still under development [47] in several cases that we discuss below; and the advent of 13 raises the possibility of working in the more general framework of homotopical categories.

We consider images of an arbitrary toric space $X_{K}$ under various functors taking values in algebraic and geometric model categories, and refer to the resulting toric objects as algebraic or geometric models for $X_{K}$. When our models are covariant, we prove that they are equivalent to the homotopy colimits of the corresponding diagrams; and when contravariant, to their homotopy limits.

An influential example is provided by Bousfield and Gugenheim's treatment [5] of the model category CDGA of commutative differential graded algebras over the rationals $\mathbb{Q}$. Their work combines with that of Sullivan [45] to show that problems of rational homotopy theory for nilpotent spaces of finite type may be solved in the homotopy category $H o(\mathrm{CDGA})$ by applying the PL-cochain functor $A_{P L}(-)$. Many 
toric spaces are simply connected CW-complexes of finite type, and their cellular cochain complexes have trivial differential. So their rationalisations retain much of the original homotopy theoretic information, and have interesting geometrical properties of their own. These may then be deduced from the contravariant algebraic models $A_{P L}\left(X_{K}\right)$, which we express as homotopy limits in CDGA. We also consider other rational models of similar power, such as differential graded coalgebras, Lie algebras, and Pontrjagin rings.

The categorical viewpoint has already motivated studies such as [35], where the rational formality of $D J(K)$ is established for every simplicial complex $K$. We extend this result below, to a class of toric spaces that includes quasitoric manifolds and the torus manifolds of [29] as special cases. By way of contrast, we note that calculations of Baskakov [2] and Denham and Suciu [12] confirm that many moment-angle complexes $\mathcal{Z}_{K}$ support non-trivial Massey products, and so cannot be formal. A further goal is to place these facts in the context of [36], where the properties of TMON as a geometric model category play an important rôle, but remain implicit. We therefore study the rational coformality 34 of $D J(K)$, which depends on the rational structure of $\Omega D J(K)$ and is verified for any flag complex $K$. Our study includes an investigation of the Lie algebra $\pi_{*}(\Omega D J(K)) \otimes_{\mathbb{Z}} \mathbb{Q}$, and is related to calculations of the Pontrjagin ring $H_{*}(\Omega D J(K) ; \mathbb{Q})$. It is particularly fascinating to try and understand how the combinatorial structure of $K$ influences the commutators and higher commutators which characterise the latter; our initial calculations in this direction have already been supplemented by those of [12].

\subsection{Contents. The contents of our sections are as follows.}

In Section 2 we recall background information on general category theory, introducing notation as we proceed. We place special emphasis on the finite categories $\operatorname{CAT}(K)$, aspects of Quillen's model category theory, and categories of CAT $(K)$ diagrams. We introduce our algebraic model categories in Section 3, where we emphasise less familiar cases by describing explicit fibrations and cofibrations. These ideas underlie Section 4 , where we outline the construction and relevant properties of homotopy limits and colimits in terms of fibrant and cofibrant replacement functors. The remaining sections are focused on applications, and Section 5 begins with straightforward examples arising from the Stanley-Reisner algebra of a simplical complex $K$ and the related space $D J(K)$. Similar examples follow in Section 6. where we discuss algebraic models for moment-angle complexes, and in Section 7. where we introduce algebraic models for quasi-toric manifolds and confirm that they are rationally formal. We then progress to loop spaces, and in Section 8 we establish algebraic analogues of our geometric model [36] for $\Omega D J(K)$ as a homotopy colimit of topological monoids. We specialise to flag complexes $K$ in Section 9. by proving that the rational Pontrjagin ring of $\Omega D J(K)$ is isomorphic to the quadratic dual of the Stanley-Reisner algebra $\mathbb{Q}[K]$ and that $D J(K)$ is coformal. In our concluding Section 10 we give additional examples concerning the cobar construction and higher commutators in Pontrjagin rings.

1.4. Algebraic Conventions. So far as general algebraic notation is concerned, we work over an arbitrary commutative ring $R$, usually indicated by means of a subscript. In many situations $R$ is restricted to the rational numbers, and in that case only we omit the subscript for reasons of notational clarity. 
We consider finite sets $W$ of generators $w_{1}, \ldots, w_{m}$, which are graded over the non-negative integers by a dimension function $\left|w_{j}\right|$, for $1 \leq j \leq m$. We write the graded tensor $R$-algebra on $W$ as $T_{R}\left(w_{1}, \ldots, w_{m}\right)$, and use the abbreviation $T_{R}(W)$ whenever possible. Its symmetrisation $S_{R}(W)$ is the graded commutative $R$-algebra generated by $W$. If $U, V \subseteq W$ are the subsets of odd and even grading respectively, then $S_{R}(W)$ is the tensor product of the exterior algebra $\wedge_{R}(U)$ and the polynomial algebra $P_{R}(V)$. It is also convenient to denote the free graded Lie algebra on $W$ and its commutative counterpart by $F L_{R}(W)$ and $C L_{R}(W)$ respectively; the latter is nothing more than a free $R$-module.

We adapt this notation to subsets $\alpha \subseteq W$ by writing $T_{R}(\alpha), S_{R}(\alpha), \wedge_{R}(\alpha)$, $F L_{R}(\alpha)$, and $C L_{R}(\alpha)$ as appropriate. Identifying $\alpha$ with its characteristic function then allows us to denote the square-free monomial $\prod_{\alpha} w_{i}$ by $w_{\alpha}$ in $S_{R}(\alpha) \leq S_{R}(W)$.

Almost all of our graded algebras have finite type, leading to a natural coalgebraic structure on their duals. We write the free tensor coalgebra on $W$ as $T_{R}\langle W\rangle$; it is isomorphic to $T_{R}(W)$ as $R$-modules, and its diagonal is given by

$$
\delta\left(w_{j_{1}} \otimes \cdots \otimes w_{j_{r}}\right)=\sum_{k=0}^{r}\left(w_{j_{1}} \otimes \cdots \otimes w_{j_{k}}\right) \otimes\left(w_{j_{k+1}} \otimes \cdots \otimes w_{j_{r}}\right) .
$$

The submodule $S_{R}\langle W\rangle$ of symmetric elements $\left(w_{i} \otimes w_{j}+(-1)^{\left|w_{i}\right|\left|w_{j}\right|} w_{j} \otimes w_{i}\right.$, for example) is the graded cocommutative $R$-coalgebra cogenerated by $W$.

Given $W$, we may sometimes define a differential by denoting the set of elements $d w_{1}, \ldots, d w_{m}$ by $d W$. The differential lowers or raises gradings by 1 , depending on whether it is homological or cohomological respectively. For example, we write the free differential graded algebra on a single generator $w$ of positive dimension as $T_{R}(w, d w)$; the notation is designed to reinforce the fact that its underlying algebra is the tensor $R$-algebra on elements $w$ and $d w$. Similarly, $T_{R}\langle w, d w\rangle$ is the free differential graded coalgebra on $w$. For further information on differential graded coalgebras, 26] remains a valuable source.

1.5. Acknowledgements. Since 2001, we have benefitted greatly from the advice and encouragement of many colleagues, particularly John Greenlees, Dietrich Notbohm, Brooke Shipley, and most notably, Rainer Vogt. The second-named author is especially grateful to the organisers of the Osaka conference for the opportunity to present our work there; he is also responsible for several long delays in completing the latex file, and offers his apologies to all those colleagues who were promised a final version in 2002 .

\section{Diagrams And Model CATEgories}

In this section we introduce aspects of category theory that are directly relevant to the study of toric spaces. We begin by recalling the finite category $\operatorname{CAT}(K)$, associated to an arbitrary simplicial complex $K$. For global purposes we turn to the notion of a Quillen model category, and outline its relevance to rational homotopy theory. The two are interwoven in the study of categories of diagrams, whose terminology and notation we introduce as we proceed. For more complete background information we refer to the books of Hirschhorn [23] and Hovey [25].

We define our simplicial complexes $K$ on a graded set $V$ of vertices $v_{1}, \ldots, v_{m}$, each of which has dimension 2. So $K$ is a collection of faces $\sigma \subseteq V$, closed under the formation of subsets and including the empty face $\varnothing$. Every face determines two 
particular subcomplexes of $K$, namely the simplex $\Delta(\sigma)$, and its boundary $\partial(\sigma)$; the former consists of all faces $\tau \subseteq \sigma$, and the latter is obtained by omitting the maximal face $\sigma$. For algebraic purposes, we occasionally prefer the vertices to have grading 1 , in which case we may replace $V$ by $U$ and $v_{i}$ by $u_{i}$ for emphasis.

We shall work with the following combinatorial categories, noting that $\Delta$ is small, and $\operatorname{CAT}(K)$ is small and finite.

\section{Notation 2.1.}

- SET: sets and functions;

- $\Delta$ : finite ordinals and nondecreasing functions;

- CAT $(K)$ : faces of a finite simplicial complex $K$, and their inclusions.

Given a small category A and an arbitrary category R, a covariant functor $D: \mathrm{A} \rightarrow \mathrm{R}$ is known as an A-diagram in R. Such diagrams are themselves the objects of a diagram category $[\mathrm{A}, \mathrm{R}]$, whose morphisms are natural transformations. When $\mathrm{A}$ is $\Delta^{o p}$, the diagrams are precisely the simplicial objects in $\mathrm{R}$, and are written as $D_{\bullet}$; the object $D(n)$ is abbreviated to $D_{n}$ for every $n \geq 0$, and forms the $n$-simplices of $D_{\mathbf{0}}$. Motivated by the example SSET of simplicial sets, we may abbreviate the diagram category to SR in this case only.

We may interpret every object $r$ of $\mathrm{R}$ as a constant A-diagram, and so define the constant functor $\kappa: \mathrm{R} \rightarrow[\mathrm{A}, \mathrm{R}]$. Whenever $\kappa$ admits a right or left adjoint $[\mathrm{A}, \mathrm{R}] \rightarrow \mathrm{R}$, it is known as the limit or colimit functor respectively.

For any object $r$ of $\mathrm{R}$, the objects of the overcategory $\mathrm{R} \downarrow r$ are morphisms $f: q \rightarrow r$, and the morphisms are the corresponding commutative triangles; the full subcategory $\mathrm{R} \Downarrow r$ is given by restricting attention to non-identities $f$. Similarly, the objects of the undercategory $r \downarrow \mathrm{R}$ are morphisms $f: r \rightarrow s$, and the morphisms are the corresponding triangles; $r \Downarrow \mathrm{R}$ is given by restriction to the non-identities. $\operatorname{In} \operatorname{CAT}(K)$ for example, we have that

$$
\operatorname{CAT}(K) \downarrow \sigma=\operatorname{CAT}(\Delta(\sigma)) \quad \text { and } \quad \operatorname{CAT}(K) \Downarrow \sigma=\operatorname{CAT}(\partial(\sigma))
$$

for any face $\sigma$. As usual, we write $\mathrm{R}(r, s)$ for the set of morphisms $r \rightarrow s$ in R.

A model category $\mathrm{MC}$ is closed with respect to the formation of certain limits and colimits, and contains three distinguished subcategories, whose morphisms are weak equivalencies e, fibrations $f$, and cofibrations $g$ respectively. Unless otherwise stated, these letters denote such morphisms henceforth. A fibration or cofibration is acyclic whenever it is also a weak equivalence. The three subcategories satisfy certain axioms, for which we follow Hirschhorn 23, Definition 7.1.3]; these strengthen Quillen's original axioms for a closed model category 38 in two minor but significant ways. Firstly, we demand closure with respect to small limits and colimits, whereas Quillen insists only that they be finite. Secondly, we demand that every morphism $h$ should factorise functorially as

$$
h=f \cdot g=f^{\prime} \cdot g^{\prime}
$$

for some acyclic $f$ and $g^{\prime}$, whereas Quillen insists only that such factorisations exist. When using results of pioneering authors such as Bousfield and Gugenheim [5] and Quillen [39], we must take account of these differences.

The axioms imply that initial and terminal objects $\circ$ and $*$ exist in MC, and that MC $\downarrow M$ and $M \downarrow \mathrm{MC}$ inherit model structures for any object $M$. 
An object of MC is cofibrant when the natural morphism $\circ \rightarrow M$ is a cofibration, and is fibrant when the natural morphism $M \rightarrow *$ is a fibration. A cofibrant approximation to an object $N$ is a weak equivalence $N^{\prime} \rightarrow N$ with cofibrant source, and a fibrant approximation is a weak equivalence $N \rightarrow N^{\prime \prime}$ with fibrant target. The full subcategories $\mathrm{MC}_{c}, \mathrm{MC}_{f}$ and $\mathrm{MC}_{c f}$ are defined by restricting attention to those objects of $\mathrm{MC}$ that are respectively cofibrant, fibrant, and both. When applied to $\circ \rightarrow N$ and $N \rightarrow *$, the factorisations (2.1) determine a cofibrant replacement functor $\omega: \mathrm{MC} \rightarrow \mathrm{MC}_{c}$, and a fibrant replacement functor $\phi: \mathrm{MC} \rightarrow \mathrm{MC}_{f}$. It follows from the definitions that $\omega$ and $\phi$ preserve weak equivalences, and that the associated acyclic fibrations $\omega(N) \rightarrow N$ and acyclic cofibrations $N \rightarrow \phi(N)$ form cofibrant and fibrant approximations respectively. These ideas are central to our definition of homotopy limits and colimits, and we shall see many examples below.

Weak equivalences need not be invertible, so objects $M$ and $N$ are deemed to be weakly equivalent if they are linked by a zig-zag $M \stackrel{e_{1}}{\longrightarrow} \cdots \stackrel{e_{n}}{\longleftarrow} N$ in $\mathrm{MC}$; this is the smallest equivalence relation generated by the weak equivalences. An important consequence of the axioms is the existence of a localisation functor $\gamma: \mathrm{MC} \rightarrow \mathrm{Ho}(\mathrm{MC})$, such that $\gamma(e)$ is an isomorphism in the homotopy category $H o(\mathrm{MC})$ for every weak equivalence. Here $H o(\mathrm{MC})$ has the same objects as $\mathrm{MC}$, and is equivalent to a category whose objects are those of $\mathrm{MC}_{c f}$, but whose morphisms are homotopy classes of morphisms between them. In $\mathrm{MC}_{c f}$, homotopy is an equivalence relation defined by means of cylinder or path objects.

Any functor $F$ of model categories that preserves weak equivalences necessarily induces $H o(F)$ on their homotopy categories, although weaker conditions suffice. Examples of the former include

$$
H o(\omega): H o(\mathrm{MC}) \rightarrow H o\left(\mathrm{MC}_{c}\right) \text { and } H o(\phi): H o(\mathrm{MC}) \rightarrow H o\left(\mathrm{MC}_{f}\right) .
$$

Such functors often occur as adjoint pairs

$$
F: \mathrm{MB} \rightleftarrows \mathrm{MC}: G,
$$

where $F$ is left Quillen if it preserves cofibrations and acyclic cofibrations, and $G$ is right Quillen if it preserves fibrations and acyclic fibrations. Either of these implies the other, leading to the notion of a Quillen pair $(F, G)$; then Ken Brown's Lemma [23. Lemma 7.7.1] applies to show that $F$ and $G$ preserve all weak equivalences on $\mathrm{MB}_{c}$ and $\mathrm{MC}_{f}$ respectively. So they may be combined with (2.2) to produce an adjoint pair of derived functors

$$
L F: H o(\mathrm{MB}) \rightleftarrows H o(\mathrm{MC}): R G,
$$

which are equivalences of the homotopy categories (or certain of their full subcategories) in favourable cases.

Our first examples of model categories are geometric, as follows.

\section{Notation 2.2.}

- TOP: pointed $k$-spaces and continuous maps [46];

- TMON: topological monoids and continuous homomorphisms.

We assume that topological monoids are $k$-spaces and are pointed by their identities, so that TMON is a subcategory of TOP. The standard model structure for TOP is described in detail by Hovey [25, Theorem 2.4.23]; weak equivalences induce isomorphisms of homotopy groups, fibrations are Serre fibrations, and cofibrations obey the left lifting property with respect to acyclic fibrations. The model structure 
for TMON is originally due to Schwänzl and Vogt [1], and may also be deduced from Schwede and Shipley's theory [42] of monoids in monoidal model categories; weak equivalences and fibrations are those homomorphisms which are weak equivalences and fibrations in TOP, and cofibrations obey the appropriate lifting property.

Our algebraic categories are defined over arbitrary commutative rings $R$, but tend only to acquire model structures when $R$ is a field of characteristic zero. If $R=\mathbb{Q}$, and in this case only, we omit the subscript from the notation.

\section{Notation 2.3.}

- $\mathrm{CH}_{R}$ and $\mathrm{COCH}_{R}$ : augmented chain and cochain complexes;

- $\mathrm{CDGA}_{R}$ : commutative augmented differential graded algebras, with cohomology differential;

- $\mathrm{DGA}_{R}$ : augmented differential graded algebras, with homology differential;

- $\mathrm{CDGC}_{R}$ : cocommutative supplemented differential graded coalgebras, with homology differential;

- $\mathrm{DGC}_{R}$ : supplemented differential graded coalgebras, with homology differential;

- DGL: differential graded Lie algebras over $\mathbb{Q}$, with homology differential.

For any model structure on these categories, weak equivalences are the quasiisomorphisms, which induce isomorphisms in homology or cohomology. The fibrations and cofibrations are described in Section 3 below. The augmentations and supplementations act as algebraic analogues of basepoints.

We reserve the notation AMC for any of the categories 2.3. and assume that objects are graded over the non-negative integers, and therefore connective; for $i \geq 0$, we denote the full subcategory of $i$-connected objects by $\mathrm{AMC}_{i}$. In order to emphasise the differential, we may sometimes display an object $M$ as $(M, d)$. The homology or cohomology group $H(M, d)$ is also an $R$-module, and inherits all structure on $M$ except for the differential. Nevertheless, we may interpret any graded algebra, coalgebra or Lie algebra as an object of the corresponding differential category, by imposing $d=0$.

Definition 2.4. An object $(M, d)$ is formal in AMC whenever there exists a zig-zag of quasi-isomorphisms

$$
(M, d)=M_{1} \stackrel{\simeq}{\longrightarrow} \cdots \stackrel{\simeq}{\longleftarrow} M_{k}=(H(M), 0) .
$$

Formality only has meaning in an algebraic model category.

Sullivan's approach to rational homotopy theory is based on the PL-cochain functor $A_{P L}$ : TOP $\rightarrow$ CDGA. Following [16], $A_{P L}(X)$ is defined as $A^{*}\left(S_{\bullet} X\right)$, where $S \bullet(X)$ denotes the total singular complex of $X$ and $A^{*}$ : SSET $\rightarrow$ CDGA is the polynomial de Rham functor of [5]. The PL-de Rham Theorem yields a natural isomorphism $H\left(A_{P L}(X)\right) \rightarrow H^{*}(X, \mathbb{Q})$, so $A_{P L}(X)$ provides a commutative replacement for rational singular cochains, and $A_{P L}$ descends to homotopy categories. Bousfield and Gugenheim describe its derived functor in terms of minimal models, and prove that it restricts to an equivalence of appropriate full subcategories of $\mathrm{Ho}$ (TOP) and $H o(\mathrm{CDGA})$. In other words, it provides a contravariant algebraic model for the rational homotopy theory of well-behaved spaces.

Quillen's approach involves the homotopy groups $\pi_{*}(\Omega X) \otimes_{\mathbb{Z}} \mathbb{Q}$, which form the rational homotopy Lie algebra of $X$ under the Samelson product. He constructs a 
covariant functor $Q: \mathrm{TOP}_{1} \rightarrow \mathrm{DGL}_{0}$, and a natural isomorphism

$$
H(Q(X)) \stackrel{\cong}{\longrightarrow} \pi_{*}(\Omega X) \otimes_{\mathbb{Z}} \mathbb{Q} .
$$

for any simply connected $X$. He concludes that $Q$ passes to an equivalence of homotopy categories; in other words, its derived functor provides a covariant algebraic model for the rational homotopy theory of simply connected spaces.

The two approaches are Eckmann-Hilton dual, but the details are subtle. Each has enabled important calculations, leading to the solution of significant geometric problems. For examples, and further details, we refer readers to [16].

Definition 2.4 is consistent with standard terminology, which describes a topological space $X$ as formal when $A_{P L}(X)$ is formal in CDGA [45], and coformal when $Q(X)$ is formal in DGL [34. In particular, $X$ is formal whenever there exists a geometric procedure for making a multiplicative choice of cocycle to represent each cohomology class; this yields a quasi-isomorphism $H^{*}(X, \mathbb{Q}) \rightarrow A_{P L}(X)$, and applies to spaces such as $\mathbb{C} P^{\infty}$.

The importance of categories of simplicial objects is due in part to the structure of the indexing category $\Delta^{o p}$. Every object $(n)$ has degree $n$, and every morphism may be factored uniquely as a composition of morphisms that raise and lower degree. These properties are formalised in the notion of a Reedy category A, which admits generating subcategories $\mathrm{A}_{+}$and $\mathrm{A}_{-}$whose non-identity morphisms raise and lower degree respectively. The diagram category $[\mathrm{A}, \mathrm{MC}]$ then supports a canonical model structure of its own [23, Theorem 15.3.4]. By duality, $\mathrm{A}^{o p}$ is also Reedy, with $\left(\mathrm{A}^{o p}\right)_{+}=\left(\mathrm{A}_{-}\right)^{o p}$ and vice-versa. A simple example is provided by $\mathrm{CAT}(K)$, whose degree function assigns the dimension $|\sigma|-1$ to each face $\sigma$ of $K$. So CAT $+(K)$ is the same as $\mathrm{CAT}_{(K)}$, and $\mathrm{CAT}_{-}(K)$ consists entirely of identities.

In the Reedy model structure on $[\mathrm{CAT}(K), \mathrm{MC}]$, weak equivalences $e: C \rightarrow D$ are given objectwise, in the sense that $e(\sigma): C(\sigma) \rightarrow D(\sigma)$ is a weak equivalence in MC for every face $\sigma$ of $K$. Fibrations are also objectwise. To describe the cofibrations, we restrict $C$ and $D$ to the overcategories CAT $(\partial(\sigma))$, and write $L_{\sigma} C$ and $L_{\sigma} D$ for their respective colimits. So $L_{\sigma}$ is the latching functor of [25], and $g: C \rightarrow D$ is a cofibration precisely when the induced maps

$$
C(\sigma) \amalg_{L_{\sigma} C} L_{\sigma} D \longrightarrow D(\sigma)
$$

are cofibrations in MC for all faces $\sigma$. Thus $D: \operatorname{CAT}(K) \rightarrow$ MC is cofibrant when every canonical map colim $D(\partial(\sigma)) \rightarrow D(\sigma)$ is a cofibration.

In the dual model structure on $\left[\mathrm{CAT}^{o p}(K), \mathrm{MC}\right]$, weak equivalences and cofibrations are given objectwise. To describe the fibrations, we restrict $C$ and $D$ to the undercategories $\mathrm{CAT}^{o p}(\partial(\sigma))$, and write $M_{\sigma} C$ and $M_{\sigma} D$ for their respective limits. So $M_{\sigma}$ is the matching functor of [25], and $f: C \rightarrow D$ is a fibration precisely when the induced maps

$$
C(\sigma) \longrightarrow D(\sigma) \times_{M_{\sigma} D} M_{\sigma} C
$$

are fibrations in MC for all faces $\sigma$. Thus $C: \mathrm{CAT}^{o p}(K) \rightarrow$ MC is fibrant when every canonical map $C(\sigma) \rightarrow \lim C(\partial(\sigma))$ is a fibration.

The axioms for a model category are actually self-dual, in the sense that any general statement concerning fibrations, cofibrations, limits, and colimits is equivalent to the statement in which they are replaced by cofibrations, fibrations, colimits, and limits respectively. In particular, $\mathrm{MC}^{o p}$ always admits a dual model structure. 


\section{Algebraic model CAtegories}

In this section we give further details of the algebraic model categories of 2.3. We describe the fibrations and cofibrations in each category, comment on the status of the strengthened axioms, and give simple examples in less familiar cases. We also discuss two important adjoint pairs.

3.1. Chain and cochain complexes. The existence of a model structure on categories of chain complexes was first proposed by Quillen 38, whose view of homological algebra as homotopy theory in $\mathrm{CH}_{R}$ was a crucial insight. Variations involving bounded and unbounded complexes are studied by Hovey [25], for example. In $\mathrm{CH}_{R}$, we assume that the fibrations are epimorphic in positive degrees and the cofibrations are monomorphic with degree-wise projective cokernel [14]. In particular, every object is fibrant.

The existence of limits and colimits is assured by working dimensionwise, and functoriality of the factorisations (2.1) follows automatically from the fact that $\mathrm{CH}_{R}$ is cofibrantly generated [25, Chapter 2].

Tensor product of chain complexes invests $\mathrm{CH}_{R}$ with the structure of a monoidal model category, as defined by Schwede and Shipley [42]. The Dold-Kan correspondence confirms that the normalisation functor $N: \mathrm{SMOD}_{R} \rightarrow \mathrm{CH}_{R}$ is an equivalence of categories [20, §3], and one of a Quillen pair. Being a simplicial model category [23, SMOD $_{R}$ is more amenable to various homotopy theoretic constructions.

Model structures on $\mathrm{COCH}_{R}$ are established by analogous techniques. It is usual to assume that the fibrations are epimorphic with degree-wise injective kernel, and the cofibrations are monomorphic in positive degrees. Then every object is cofibrant. There is an alternative structure based on projectives, but we shall only refer to the rational case so we ignore the distinction. Tensor product turns $\mathrm{COCH}_{R}$ into a monoidal model category.

3.2. Commutative differential graded algebras. We consider commutative differential graded algebras over $\mathbb{Q}$ with cohomology differentials, so they are commutative monoids in COCH. A model structure on CDGA was first defined in this context by Bousfield and Gugenheim 5, and has played a significant role in the theoretical development of rational homotopy theory ever since. The fibrations are epimorphic, and the cofibrations are determined by the appropriate lifting property; some care is required to identify sufficiently many explicit cofibrations.

Limits in CDGA are created in the underlying category $\mathrm{COCH}$ and endowed with the natural algebra structure, whereas colimits exist because CDGA has finite coproducts and filtered colimits. The proof of the factorisation axioms in [5] is already functorial.

By way of example, we note that the product of algebras $A$ and $B$ is their augmented sum $A \oplus B$, defined by pulling back the diagram of augmentations

$$
A \stackrel{\varepsilon_{A}}{\longrightarrow} \mathbb{Q} \stackrel{\varepsilon_{B}}{\longleftarrow} B
$$

in $\mathrm{COCH}$ and imposing the standard multiplication on the result. The coproduct is their tensor product $A \otimes B$ over $\mathbb{Q}$. Examples of cofibrations include extensions of the form $A \rightarrow(A \otimes S(w), d)$, determined by cocycles $z$ in $A$; such an extension is defined by pushing out the diagram

$$
A \stackrel{h}{\longleftarrow} S(x) \stackrel{j}{\longrightarrow} S(w, d w),
$$


where $h(x)=z$ and $j(x)=d w$. So the differential on $A \otimes S(w)$ is given by

$$
d(a \otimes 1)=d_{A} a \otimes 1 \quad \text { and } d(1 \otimes w)=z \otimes 1 .
$$

This illustrates the fact that the pushout of a cofibration is a cofibration. A larger class of cofibrations $A \rightarrow A \otimes S(W)$ is given by iteration, for any set $W$ of positive dimensional generators corresponding to cocycles in $A$.

The factorisations (2.1) are only valid over fields of characteristic 0 , so the model structure does not extend to $\mathrm{CDGA}_{R}$ for arbitrary rings $R$.

3.3. Differential graded algebras. Our differential graded algebras have homology differentials, and are the monoids in $\mathrm{CH}_{R}$. A model category structure in $\mathrm{DGA}_{R}$ is therefore induced by applying Quillen's path object argument, as in 42]; a similar structure was first proposed by Jardine, 27] (albeit with cohomology differentials), who proceeds by modifying the methods of [5. Fibrations are epimorphisms, and cofibrations are determined by the appropriate lifting property.

Limits are created in $\mathrm{CH}_{R}$, whereas colimits exist because $\mathrm{DGA}_{R}$ has finite coproducts and filtered colimits. Functoriality of the factorisations follows by adapting the proofs of [5], and works over arbitrary $R$.

For example, the coproduct of algebras $A$ and $B$ is the free product $A \star B$, formed by factoring out an appropriate differential graded ideal 27] from the free tensor algebra $T_{R}(A \otimes B)$ on the chain complex $A \otimes B$. Examples of cofibrations include the extensions $A \rightarrow\left(A \star T_{R}(w), d\right)$, determined by cycles $z$ in $A$. By analogy with the commutative case, such an extension is defined by pushing out the diagram

$$
A \stackrel{h}{\longleftarrow} T_{R}(x) \stackrel{j}{\longrightarrow} T_{R}(w, d w),
$$

where $h(x)=z$ and $j(x)=d w$. The differential on $A \star T_{R}(w)$ is given by

$$
d(a \star 1)=d_{A} a \star 1 \quad \text { and } \quad d(1 \star w)=z \star 1 .
$$

Further cofibrations $A \rightarrow A \star T_{R}(W)$ arise by iteration, for any set $W$ of positive dimensional generators corresponding to cycles in $A$.

3.4. Cocommutative differential graded coalgebras. The cocommutative comonoids in $\mathrm{CH}_{R}$ are the objects of $\mathrm{CDGC}_{R}$, and the morphisms preserve comultiplication. The model structure is defined only over fields of characteristic 0 ; in view of our applications, we shall restrict attention to the case $\mathbb{Q}$. In practice, we interpret $\mathrm{CDGC}$ as the full subcategory $\mathrm{CDGC}_{0}$ of connected objects $C$, which are necessarily supplemented. Model structure was first defined on the category $\mathrm{CDGC}_{1}$ of simply connected rational cocommutative coalgebras by Quillen 39], and refined to $\mathrm{CDGC}_{0}$ by Neisendorfer [33. The cofibrations are monomorphisms, and the fibrations are determined by the appropriate lifting property.

Limits exist because CDGC has finite products and filtered limits, whereas colimits are created in $\mathrm{CH}$, and endowed with the natural coalgebra structure. Functoriality of the factorisations again follows by adapting the proofs of [5].

For example, the product of coalgebras $C$ and $D$ is their tensor product $C \otimes D$ over $\mathbb{Q}$. The coproduct is their supplemented sum, given by pushing out the diagram of supplementations

$$
C \stackrel{\delta_{C}}{\longleftarrow} \mathbb{Q} \stackrel{\delta_{D}}{\longrightarrow} D
$$

in $\mathrm{CH}$ and imposing the standard comultiplication on the result. Examples of fibrations include the projections $(C \otimes S\langle d w\rangle, d) \rightarrow C$, which are determined by cycles 
$z$ in $C$ and obtained by pulling back diagrams

$$
C \stackrel{h}{\longrightarrow} S\langle x\rangle \stackrel{p}{\longleftarrow} S\langle w, d w\rangle,
$$

where $p(w)=x, p(d w)=0$ and $h(z)=x$. The differential on $C \otimes S\langle d w\rangle$ satisfies

$$
d(z \otimes 1)=1 \otimes d w \quad \text { and } \quad d(1 \otimes d w)=0 .
$$

This illustrates the fact that the pullback of a fibration is a fibration. Further fibrations $C \otimes S\langle d W\rangle \rightarrow C$ are given by iteration, for any set $W$ of generators corresponding to elements of degree $\geq 2$ in $C$.

3.5. Differential graded coalgebras. Model structures on more general categories of differential graded coalgebras have been publicised by Getzler and Goerss $[19$, who also work over a field. Once more, we restrict attention to $\mathbb{Q}$. The objects of DGC are comonoids in $\mathrm{CH}$, and the morphisms preserve comultiplication. The cofibrations are monomorphisms, and the fibrations are determined by the appropriate lifting property.

Limits exist because DGC has finite products and filtered limits, and colimits are created in $\mathrm{CH}$. Functoriality of factorisations follows from the fact that the model structure is cofibrantly generated.

For example, the product of coalgebras $C$ and $D$ is the cofree product $C \star D$ 19. Their coproduct is the supplemented sum, as in the case of CDGC. Examples of fibrations include the projections $(C \star T\langle d w\rangle, d) \rightarrow C$, which are determined by cycles $z$ in $C$ and obtained by pulling back diagrams

$$
C \stackrel{h}{\longrightarrow} T\langle x\rangle \stackrel{p}{\longleftarrow} T\langle w, d w\rangle,
$$

where $p(w)=x, p(d w)=0$ and $h(z)=x$. The differential on $C \star T\langle d w\rangle$ satisfies

$$
d(z \star 1)=1 \star d w \quad \text { and } \quad d(1 \star d w)=0 .
$$

3.6. Differential graded Lie algebras. For our purposes here, a differential graded Lie algebra $L$ is a chain complex in $\mathrm{CH}$, equipped with a bracket morphism $L \otimes L \rightarrow L$ satisfying signed versions of the antisymmetry and Jacobi identities. Differential graded Lie algebras over $\mathbb{Q}$ are the objects of DGL, and their homomorphisms are the morphisms. Quillen [39] originally defined a model structure on the subcategory DGL 1 of reduced objects, which was extended to DGL by Neisendorfer 33. Fibrations are epimorphisms, and cofibrations are determined by the appropriate lifting property.

Limits are created in $\mathrm{CH}$, whereas colimits exist because DGL has finite coproducts and filtered colimits. Functoriality of the factorisations follows by adapting the proofs of 33 .

For example, the product of Lie algebras $L$ and $M$ is their product $L \oplus M$ as chain complexes, with the induced bracket structure. Their coproduct is the free product $L \star M$, obtained by factoring out an appropriate differential graded ideal from the free Lie algebra $F L(L \otimes M)$ on the chain complex $L \otimes M$. Examples of cofibrations include the extensions $L \rightarrow(L \star F L(w), d)$, which are determined by cycles $z$ in $L$ and obtained by pushing out diagrams

$$
L \stackrel{h}{\longleftarrow} F L(x) \stackrel{j}{\longrightarrow} F L(w, d w),
$$

where $h(x)=z$ and $j(x)=d w$. The differential on $L \star F(w)$ is given by

$$
d(l \star 1)=d_{L} l \star 1 \quad \text { and } \quad d(1 \star w)=z \star 1 .
$$


For historical reasons, a differential graded Lie algebra $L$ is said to be coformal whenever it is formal in DGL.

3.7. Adjoint pairs. Following Moore [32, 26], we consider the classifying functor $B_{*}$ and the loop functor $\Omega_{*}$ as an adjoint pair

$$
\Omega_{*}: \mathrm{DGC}_{0, R} \rightleftarrows \mathrm{DGA}_{R}: B_{*} .
$$

For any object $A$ of $\mathrm{DGA}_{R}$, the classifying coalgebra $B_{*} A$ agrees with Eilenberg and Mac Lane's normalised bar construction as objects of $\mathrm{CH}_{R}$. For any object $C$ of $\operatorname{DGC}_{0, R}$, the loop algebra $\Omega_{*} C$ is given by the tensor algebra $T_{R}\left(s^{-1} \bar{C}\right)$ on the desuspended $R$-module $\bar{C}=\operatorname{Ker}(\varepsilon: C \rightarrow R)$, and agrees with Adams's cobar construction [1] as objects of $\mathrm{CH}_{R}$. The graded homology algebra $H\left(\Omega_{*} C\right)$ is denoted by $\operatorname{Cotor}_{C}(R, R)$, and is isomorphic to the Pontrjagin ring $H_{*}(\Omega X ; R)$ when $C$ is the singular chain complex of a reduced CW-complex $X$.

Over $\mathbb{Q}$, the adjunction maps $C \mapsto B_{*} \Omega_{*} C$ and $\Omega_{*} B_{*} A \mapsto A$ are quasi-isomorphisms for every object $A$ and $C$. We explore the consequence of this fact further in Section 8. In particular, there is an isomorphism

$$
\operatorname{Cotor}_{C}(\mathbb{Q}, \mathbb{Q}) \cong \operatorname{Ext}_{C^{*}}(\mathbb{Q}, \mathbb{Q})
$$

of graded algebras [37, page 41], where $C^{*}$ is the graded algebra dual to $C$ and $\operatorname{Ext}_{C^{*}}(\mathbb{Q}, \mathbb{Q})$ is the rational Yoneda algebra of $C^{*}[28$.

Following Neisendorfer [33, Proposition 7.2], we consider a second pair of adjoint functors

$$
L_{*}: \mathrm{CDGC}_{0} \rightleftarrows \mathrm{DGL}: M_{*},
$$

whose derived functors induce an equivalence between $\mathrm{Ho}\left(\mathrm{CDGC}_{0}\right)$ and a certan full subcategory of $H o(\mathrm{DGL})$. This extends Quillen's original results 39 for $L_{*}$ and $M_{*}$, which apply only to simply connected coalgebras and connected Lie algebras. Given a connected cocommutative coalgebra $C$, the underlying graded Lie algebra of $L_{*} C$ is the free Lie algebra $F L\left(s^{-1} \bar{C}\right) \subset T\left(s^{-1} \bar{C}\right)$. This is preserved by the differential in $\Omega_{*} C$ because $C$ is cocommutative, thereby identifying $L_{*} C$ as the differential graded Lie algebra of primitives in $\Omega_{*} C$. The right adjoint functor $M_{*}$ may be regarded as a generalisation to differential graded objects of the standard complex for calculating the cohomology of Lie algebras. Given any $L$ in DGL the underlying cocommutative coalgebra of $M_{*} L$ is the symmetric coalgebra $C(s L)$ on the suspended vector space $L$.

\section{4. Номотору Limits AND COLImits}

The $\lim$ and colim functors $[\mathrm{A}, \mathrm{MC}] \rightarrow \mathrm{MC}$ do not generally preserve weak equivalences, and the theory of homotopy limits and colimits has been developed to remedy this deficiency. We outline their construction in this section, and discuss basic properties. The literature is still in a state of considerable flux, and we refer to Recke's thesis [40] for a comparison of several alternative treatments. Here we focus mainly on those of Recke's statements that are inspired by Hirschhorn, and make detailed appeal to [23] as necessary.

With $\operatorname{CAT}(K)$ and $\mathrm{CAT}^{o p}(K)$ in mind as primary examples, we assume throughout that A is a finite Reedy category. One additional property is especially useful. 
Definition 4.1. A Reedy category A has cofibrant constants if the constant Adiagram $M$ is Reedy cofibrant in [A, MC], for any cofibrant object $M$ of an arbitrary model category MC. Similarly, A has fibrant constants if the constant A-diagram $N$ is Reedy fibrant for any fibrant object $N$ of MC.

Note that the initial and terminal objects of $[\mathrm{A}, \mathrm{MC}]$ are the constant diagrams $\circ$ and $*$ respectively.

Lemma 4.2. The Reedy categories $\mathrm{CAT}(K)$ and $\mathrm{CAT}^{o p}(K)$ have fibrant and cofibrant constants, for every simplicial complex $K$.

Proof. Consider $\operatorname{CAT}(K)$, and any face $\sigma$. Then $\sigma \Downarrow \mathrm{CAT}_{-}(K)$ is empty, since no morphism lowers degree, whereas $\mathrm{CAT}_{+}(K) \Downarrow \sigma$ has initial object $\varnothing$, and is therefore connected. So $\operatorname{CAT}(K)$ satisfies the criteria of [23, Proposition 15.10.2] for fibrant and cofibrant constants. The result for $\mathrm{CAT}^{o p}(K)$ follows by duality.

As shown in 23. Theorem 15.10.8], a Reedy category A has fibrant and cofibrant constants if and only if the adjoint functors

$$
\kappa: \mathrm{MC} \rightleftarrows[\mathrm{A}, \mathrm{MC}]: \lim \text { and } \operatorname{colim}:[\mathrm{A}, \mathrm{MC}] \rightleftarrows \mathrm{MC}: \kappa
$$

are Quillen pairs, for every model category MC. The proof addresses the equivalent statement that $\kappa$ is both left and right Quillen. In these circumstances, it follows from Ken Brown's Lemma that lim and colim preserve weak equivalences on Reedy fibrant and Reedy cofibrant diagrams respectively.

We now apply the fibrant and cofibrant replacement functors associated to the Reedy model structure on [A, MC], and their homotopy functors (2.2).

Definition 4.3. For any Reedy category A with fibrant and cofibrant constants, and any model category MC:

(1) the homotopy colimit functor is the composition

$$
\text { hocolim: Ho }[\mathrm{A}, \mathrm{MC}] \stackrel{H o(\omega)}{\longrightarrow} H o[\mathrm{~A}, \mathrm{MC}]_{c} \stackrel{H o(\operatorname{colim})}{\longrightarrow} H o(\mathrm{MC}) \text {; }
$$

(2) the homotopy limit functor is the composition

$$
\text { holim: } H o[\mathrm{~A}, \mathrm{MC}] \stackrel{H o(\phi)}{\longrightarrow} H o[\mathrm{~A}, \mathrm{MC}]_{f} \stackrel{H o(\lim )}{\longrightarrow} H o(\mathrm{MC}) \text {. }
$$

Lemma 4.2 confirms that holim and hocolim: $H_{0}[\mathrm{CAT}(K), \mathrm{MC}] \rightarrow H_{o}(\mathrm{MC})$ are defined for any simplicial complex $K$; and similarly for $\mathrm{CAT}^{o p}(K)$.

Remark 4.4. Definition 4.3 incorporates the fact that holim and hocolim map objectwise weak equivalences of diagrams to weak equivalences in MC.

Describing explicit models for homotopy limits and colimits has been a major objective for homotopy theorists since their study was initiated by Bousfield and Kan [6. In terms of Definition 4.3] the issue is to choose fibrant and cofibrant replacement functors $\phi$ and $\omega$. Many alternatives exist, including those defined by the two-sided bar and cobar constructions of [36] or the frames of [23, §16.6], but no single description yet appears to be convenient in all cases. Instead, we accept a variety of possibilities, which are often implicit; the next few results ensure that they are as compatible and well-behaved as we need.

Proposition 4.5. Any Reedy cofibrant approximation $D^{\prime} \rightarrow D$ of diagrams induces a weak equivalence colim $D^{\prime} \rightarrow$ hocolim $D$ in $\mathrm{MC}$; and any Reedy fibrant approximation $D \rightarrow D^{\prime \prime}$ induces a weak equivalence holim $D \rightarrow \lim D^{\prime \prime}$. 
Proof. A cofibrant approximation $i: D^{\prime} \rightarrow D$ factorises as $D^{\prime} \rightarrow \omega(D) \rightarrow D$ by the lifting axiom in $[\mathrm{A}, \mathrm{MC}]$, and the left hand map is a weak equivalence by the 2 out of 3 axiom. But $D^{\prime}$ and $\omega(D)$ are cofibrant, and colim is left Quillen, so the induced map colim $D^{\prime} \rightarrow \operatorname{colim} \omega(D)$ is a weak equivalence, as required. The proof for $\lim$ is dual.

Remark 4.6. Such arguments may be strengthened to include uniqueness statements, and show that the replacements $\phi(D)$ and $\omega(D)$ are themselves unique up to homotopy equivalence over $D$ [23, Proposition 8.1.8].

Proposition 4.7. For any Reedy cofibrant diagram $D$ and fibrant diagram $E$, there are natural weak quivalences hocolim $D \rightarrow \operatorname{colim} D$ and $\lim E \rightarrow \operatorname{holim} E$.

Proof. For $D$, it suffices to apply the left Quillen functor colim to the acyclic fibration $\omega(D) \rightarrow D$. The proof for $E$ is dual.

Proposition 4.8. In any model category MC:

(1) if all three objects of a pushout diagram $D:=L \leftarrow M \rightarrow N$ are cofibrant, and either of the maps is a cofibration, then there exists a weak equivalence $\operatorname{hocolim} D \rightarrow \operatorname{colim} D$;

(2) if all three objects of a pullback diagram $E:=P \rightarrow Q \leftarrow R$ are fibrant, and either of the maps is a fibration, then there exists a weak equivalence $\operatorname{holim} E \rightarrow \lim E$.

Proof. For (1), assume that $M \rightarrow N$ is a cofibration, and that the indexing category B for $D$ has non-identity morphisms $\lambda \leftarrow \mu \rightarrow \nu$. The degree function $\operatorname{deg}(\lambda)=0$, $\operatorname{deg}(\mu)=1$, and $\operatorname{deg}(\nu)=2$ turns B into a Reedy category with fibrant constants, and ensures that $D$ is Reedy cofibrant. So Proposition 4.7 applies. If $M \rightarrow L$ is a cofibration, the corresponding argument holds by symmetry.

For (2), the proofs are dual.

Further details may be found in [23, Proposition 19.9.4].

An instructive example of cofibrant replacement is provided by the model category TOP, whose every object $X$ admits a $C W$ model. It consists of a cofibrant approximation $f: W_{X} \rightarrow X$, where $W_{X}$ is filtered by skeleta $W^{(n)}$; these are constructed inductively by the adjunction of euclidean cells, using repeated pushouts of the form $W^{(n)} \leftarrow S^{n} \rightarrow D^{n+1}$. Two weakly equivalent topological spaces have homotopy equivalent CW-models. Such models have several advantages; for example, the cellular chain complex $C e_{*}\left(W_{X}\right)$ is far more economical than the singular version $C_{*}(X)$ for computing the homology of $X$. They may, however, be difficult to make explicit, and are not functorial. A genuine cofibrant replacement functor $\omega(X) \rightarrow X$ must be constructed with care, and is defined in [14, §98], for example.

An analogous example is given by CDGA, whose every homologically connected object $A$ admits a minimal model [45. It consists of a cofibrant approximation $f: M_{A} \rightarrow A$, where $M_{A}$ is connected, is free as a commutative graded algebra, and contains a natural sequence of subalgebras that are constructed by successive pushouts of the form (3.1). It follows from these requirements that the differential of $M_{A}$ takes decomposable values only. Any two minimal models for $A$ are necessarily isomorphic, and $M_{A}$ and $M_{B}$ are isomorphic for quasi-isomorphic $A$ and $B$. So every zig-zag $A \rightarrow \cdots \leftarrow B$ in CDGA may be replaced by a diagram $A \leftarrow M \rightarrow B$, where $M$ is minimal for both $A$ and $B$. The advantage of $M_{A}$ is that it simplifies 
many calculations concerning $A$; disadvantages include the fact that it may be difficult to describe for relatively straightforward objects $A$, and that it cannot be chosen functorially. A genuine cofibrant replacement functor requires additional care, and seems first to have been made explicit in [5, §4.7].

\section{Models FOR $D J(K)$}

In this section we introduce algebraic and geometrical models for the DavisJanuszkiewicz spaces $D J(K)$. Several of the results appear in [8], [35], and [36], but our current aim is to display them in the model categorical setting; they are then more readily comparable with calculations in later sections. Analogous constructions may be made in the real case by substituting $\mathbb{Z} / 2$ for $T[11$.

Over $R$, the graded Stanley-Reisner algebra [4] of an arbitrary simplicial complex $K$ is given by the quotient

$$
R[K]=S_{R}(V) /\left(v_{\zeta}: \zeta \notin K\right),
$$

otherwise known as the face ring of $K$. Any quotient of $S_{R}(V)$ by a square-free monomial ideal is the Stanley-Reisner algebra of some simplicial complex.

The Stanley-Reisner coalgebra $R\langle K\rangle$ is the graded dual of $R[K]$, and is less wellknown. To aid its description, we write $K$ • for the simplicial set generated by $K$ [30. Example 1.4], whose non-degenerate simplices are the faces of $K$. Then $R\langle K\rangle$ is the free $R$-module on generators $v\langle\sigma\rangle$, as $\sigma$ varies over the simplices of $K_{\bullet}$. The coproduct takes the form

$$
\delta v\langle\sigma\rangle=\sum_{\sigma=\tau \sqcup \tau^{\prime}} v\langle\tau\rangle \otimes v\left\langle\tau^{\prime}\right\rangle,
$$

where the sum ranges over all partitions of $\sigma$ into subsimplices $\tau$ and $\tau^{\prime}$. By construction, the elements $v\langle\sigma\rangle$ form the basis dual to the generating monomials $v_{\sigma}$ of $R[K]$.

The exterior face ring $R_{\wedge}[K]$ is defined by analogy with (5.1), on the 1-dimensional vertices $U$. So long as $\frac{1}{2} \in R$, its dual $R^{\wedge}\langle K\rangle$ is the free $R$-module on generators $u\langle\sigma\rangle$, as $\sigma$ varies over the faces of $K$; the diagonal is also given by (5.2).

In the context of algebraic toric objects, Stanley-Reisner algebras and coalgebras may also be described as limits and colimits of $\operatorname{CAT}(K)$ diagrams 36. We invest them with zero differential, and work in the categories $\mathrm{DGA}_{R}$ and $\mathrm{DGC}_{R}$ respectively. In $\mathrm{DGA}_{R}$ we define the $\mathrm{CAT}^{o p}(K)$-diagram $S_{K}(V)$, whose value on $\tau \supseteq \sigma$ is the projection $S_{R}(\tau) \rightarrow S_{R}(\sigma)$; and in $\mathrm{DGC}_{R}$ we define the CAT $(K)$-diagram $S^{K}\langle V\rangle$, whose value on $\sigma \subseteq \tau$ is the inclusion $S_{R}\langle\sigma\rangle \rightarrow S_{R}\langle\tau\rangle$ of coalgebras. Then there are isomorphisms

$$
R[K] \stackrel{\cong}{\longrightarrow} \lim ^{\mathrm{CDGA}_{R}} S_{K}(V) \text { and } \operatorname{colim}^{\mathrm{CDGC}_{R}} S^{K}\langle V\rangle \stackrel{\cong}{\longrightarrow} R\langle K\rangle
$$

(here and below the superscript in the notation for limit and colimit refers to the target category). The limits and colimits of (5.3) are created in $\mathrm{CH}_{R}$ and the additional algebraic structure is superimposed; so they may equally well be taken over DGA $R$ and $\mathrm{DGC}_{R}$.

In the rational case, CDGA and CDGC admit the model structures of subsections 3.2 and 3.4 so the corresponding homotopy limits and homotopy colimits exist. 
Since the diagrams $S_{K}(V)$ and $S^{K}\langle V\rangle$ are Reedy fibrant and Reedy cofibrant respectively, we may rewrite (5.3) in terms of weak equivalences

$$
\mathbb{Q}[K] \stackrel{\simeq}{\longrightarrow} \operatorname{holim}^{\mathrm{CDGA}} S_{K}(V) \text { and } \operatorname{hocolim}^{\mathrm{CDGC}} S^{K}\langle V\rangle \stackrel{\simeq}{\longrightarrow} \mathbb{Q}\langle K\rangle,
$$

by applying Proposition 4.7. Similar remarks apply to the exterior cases.

In [11, Davis and Januszkiewicz introduce the space now known as $D J(K)$ by means of a Borel construction. It is designed to ensure that the cohomology ring $H^{*}(D J(K) ; \mathbb{Z})$ is isomorphic to $\mathbb{Z}[K]$, and therefore that the homology coalgebra $H_{*}(D J(K) ; \mathbb{Z})$ is isomorphic to $\mathbb{Z}\langle K\rangle$ by duality.

In 8 and [36, the $\operatorname{CAT}(K)$ diagram $B T^{K}$ is defined in TOP; its colimit

$$
\operatorname{colim} B T^{K}=\bigcup_{\sigma \in K} B T^{\sigma}
$$

is a subcomplex of $B T^{V}$, and is shown to be homotopy equivalent to $D J(K)$. Here $T^{\sigma}<T^{V}$ is the coordinate subtorus, so its classifying space $B T^{\sigma}$ is automatically a subcomplex of $B T^{V} \simeq\left(C P^{\infty}\right)^{V}$. The colimit inherits a natural cell structure from $B T^{V}$, whose cellular chain coalgebra may readily be identified with $R\langle K\rangle$ for any coefficient ring $R$. Since $B T^{K}$ is Reedy cofibrant, there is a weak equivalence hocolim $B T^{K} \rightarrow D J(K)$, by Proposition 4.7 .

Similar remarks apply to the exterior case, by replacing the circle $T$ with $\mathbb{Z}$. So the diagram $B \mathbb{Z}^{K}$ may be identified with $T^{K}$, and is also Reedy cofibrant; its colimit $D J_{\wedge}(K)$ is a subcomplex of the torus $T^{V}$, and is necessarily finite. By construction, $H^{*}\left(D J_{\wedge}(K) ; R\right)$ and $H_{*}\left(D J_{\wedge}(K) ; R\right)$ are isomorphic to $R_{\wedge}[K]$ and $R^{\wedge}\langle K\rangle$ respectively.

Sullivan's functor $A_{P L}$ usually provides more sophisticated contravariant models, in terms of rational cochain algebras. However, the space $D J(K)$ is formal 35 for every complex $K$, and we may use its minimal model to reduce the zig-zag of quasi-isomorphisms (2.4) to the form

$$
\mathbb{Q}[K] \stackrel{e_{K}}{\longleftarrow} M(D J(K)) \stackrel{\simeq}{\longrightarrow} A_{P L}(D J(K)
$$

in CDGA. In this sense, $\mathbb{Q}[K]$ is just as good a model for $D J(K)$ as $A_{P L}(D J(K))$; for a discussion of uniqueness, we refer to [35, Proposition 5.10]. Results on the coformality of $D J(K)$ are presented in Section 9 .

We summarise this section in terms of our motivating principle, that certain functors preserve homotopy limit and colimit structures on specific toric objects.

Proposition 5.1. As functors $\mathrm{TOP} \rightarrow \mathrm{CDGC}$ and $\mathrm{TOP} \rightarrow \mathrm{CDGA}$ respectively, rational homology preserves homotopy colimits and rational cohomology maps homotopy colimits to homotopy limits, on diagrams $T^{K}$ and $B T^{K}$.

Proposition 5.1 generalises immediately to the integral situation, although the model categorical framework has to be relaxed in the coalgebraic case.

\section{Models FOR $\mathcal{Z}_{K}$}

In this section we introduce algebraic and geometrical models for the moment angle complexes $\mathcal{Z}_{K}$ of [11, which form a second important class of toric spaces associated to a simplicial complex $K$.

Many applications to toric manifolds and combinatorial commutative algebra are developed in [8], where $\mathcal{Z}_{K}$ is described as the homotopy fibre of the inclusion 
$D J(K) \rightarrow B T^{V}$. As such, it is obtained by pulling back the diagram

$$
P_{K}:=D J(K) \stackrel{\subseteq}{\longrightarrow} B T^{V} \stackrel{p}{\longleftarrow} E T^{V}
$$

in TOP, and therefore by restricting the universal $T^{V}$-bundle $E T^{V} \rightarrow B T^{V}$ to $D J(K)$. So $\mathcal{Z}_{K}$ inherits a canonical $T^{V}$-action, and there exists a weak equivalence $\mathcal{Z}_{K} \rightarrow \operatorname{holim} P_{K}$ by Proposition 4.8(2).

Alternatively, $\mathcal{Z}_{K}$ may be identified with the subcomplex $\bigcup_{\sigma \in K} D_{\sigma}$ of the standard unit $2 m$-disk $\left(D^{2}\right)^{V} \subset \mathbb{C}^{V}$, where

$$
D_{\sigma}=\left\{\left(z_{1}, \ldots, z_{m}\right) \in\left(D^{2}\right)^{V}:\left|z_{j}\right|=1 \text { for } j \notin \sigma\right\} .
$$

Note that $D_{\sigma} \cong\left(D^{2}\right)^{s} \times T^{m-s}$ when $|\sigma|=s$, for every $0 \leq s \leq m$. A convenient cellular structure on $\mathcal{Z}_{K}$ is given by combining this description with the decomposition of $D^{2}$ into a single cell in each dimension 0,1 , and 2 . If $K$ is a triangulated sphere then $\mathcal{Z}_{K}$ is homotopy equivalent to a manifold [8, Lemma 6.13].

We consider CAT $(K)$-diagrams $D^{K}$ and $T^{V / K}$, whose values on $\sigma \subseteq \tau$ are the inclusion $D_{\sigma} \subseteq D_{\tau}$ and the quotient $T^{V} / T^{\sigma} \rightarrow T^{V} / T^{\tau}$ respectively. In particular, $\operatorname{colim} D^{K}$ is $\mathcal{Z}_{K}$. Objectwise projection induces a weak equivalence $D^{K} \rightarrow T^{V / K}$ in $[\mathrm{CAT}(K)$, TOP], whose source is Reedy cofibrant but whose target is not. Proposition 4.5 therefore determines a weak equivalence

$$
\operatorname{colim} D^{K} \stackrel{\simeq}{\longrightarrow} \operatorname{hocolim} T^{V / K},
$$

which agrees with the equivalence $\mathcal{Z}_{K} \simeq \operatorname{hocolim} T^{V / K}$ of [36, Corollary 5.4].

So we have described $\mathcal{Z}_{K}$ as holim $P_{K}$ and as hocolim $T^{V / K}$. A weak equivalence between the two is given in the categorical context by [36, Proposition 5.1], and we shall apply $A_{P L}$ to each. We employ the standard model [16, $\left.\S 15(\mathrm{c})\right]$ for the principal $T^{V}$-bundle of (6.1), given by the commutative diagram

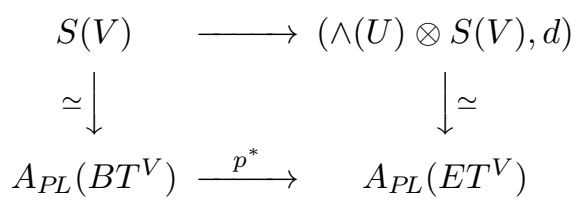

in CDGA. The differential satisfies $d u_{j}=v_{j}$ for $1 \leq j \leq m$; also, $p^{*}$ is a cofibration of the form described in Subsection 3.2, and $S(V)$ is the minimal model for $B T^{V}$.

First we apply $A_{P L}$ to (6.1), on the understanding that it does not generally convert pullbacks to pushouts [5, §3]. We obtain the CDGA-diagram

$$
P_{K}^{\prime}:=A_{P L}(D J(K)) \longleftarrow A_{P L}\left(B T^{V}\right) \longrightarrow A_{P L}\left(E T^{V}\right),
$$

which is not Reedy cofibrant.

Theorem 6.1. The algebras $A_{P L}\left(\mathcal{Z}_{K}\right)$ and hocolim $P_{K}^{\prime}$ are weakly equivalent in CDGA.

Proof. By (6.4), there is an objectwise weak equivalence mapping the diagram

$$
P_{K}^{\prime \prime}:=M(D J(K)) \stackrel{q}{\longleftarrow} S(V) \longrightarrow \wedge(U) \otimes S(V)
$$

to $P_{K}^{\prime}$. Moreover, colim $P_{K}^{\prime \prime}$ is given by $(\wedge(U) \otimes M(D J(K)), d)$, where $d u_{j}=q\left(v_{j}\right)$ for $1 \leq j \leq m$, and colim $P_{K}^{\prime}$ maps naturally to $A_{P L}\left(\mathcal{Z}_{K}\right)$; so the composition $e: \wedge(U) \otimes M(D J(K)) \rightarrow A_{P L}\left(\mathcal{Z}_{K}\right)$ is defined. Since $D J(K)$ is simply connected, $e$ is a quasi-isomorphism by [16, $\S 15(\mathrm{c})]$. 
The right hand arrow of $P_{K}^{\prime \prime}$ is a cofibration and its objects are cofibrant, so there is a quasi-isomorphism hocolim $P_{K}^{\prime \prime} \rightarrow \operatorname{colim} P_{K}^{\prime \prime}$ by Proposition 4.8(1). Furthermore, the induced map hocolim $P_{K}^{\prime \prime} \rightarrow \operatorname{hocolim} P_{K}^{\prime}$ is a quasi-isomorphism by Remark 4.4, So there is a zig-zag

$$
\operatorname{hocolim} P_{K}^{\prime} \longleftarrow \operatorname{hocolim} P_{K}^{\prime \prime} \longrightarrow A_{P L}\left(\mathcal{Z}_{K}\right)
$$

of quasi-isomorphisms in CDGA, as required.

In order to apply $A_{P L}$ to (6.3), we consider $\mathrm{CAT}^{o p}(K)$-diagrams $\wedge(U) \otimes S_{K}(V)$ and $\wedge_{U / K}$ in CDGA. The values of the former on $\tau \supseteq \sigma$ are the quotient maps

$$
(\wedge(U) \otimes S(\tau), d) \longrightarrow(\wedge(U) \otimes S(\sigma), d),
$$

where $d$ is defined on $\wedge(U) \otimes S(\sigma)$ by $d u_{j}=v_{j}$ for $u_{j} \in \sigma$, and 0 otherwise; the values of the latter are the monomorphisms $\wedge\left(u_{i} \notin \tau\right) \rightarrow \wedge\left(u_{i} \notin \sigma\right)$ in CDGA. Objectwise projection induces a weak equivalence

$$
\wedge(U) \otimes S_{K}(V) \stackrel{\simeq}{\longrightarrow} \wedge_{U / K}
$$

in $\left[\mathrm{CAT}^{o p}(K), \mathrm{CDGA}\right]$, whose source is Reedy fibrant but whose target is not.

Theorem 6.2. The algebras $A_{P L}\left(\mathcal{Z}_{K}\right)$ and holim $\wedge_{U / K}$ are weakly equivalent in CDGA.

Proof. Choosing representative cocycles for generators of $H^{1}\left(T^{V} ; \mathbb{Q}\right)$ yields compatible quasi-isomorphisms $\wedge\left(u_{i} \notin \sigma\right) \rightarrow A_{P L}\left(D_{\sigma}\right)$, as $\sigma$ ranges over the faces of $K$. These define a weak equivalence $\wedge_{U / K} \rightarrow A_{P L} \circ D^{K}$ of $\mathrm{CAT}^{o p}(K)$-diagrams, which combines with (6.6) to give a weak equivalence

$$
\wedge(U) \otimes S_{K}(V) \stackrel{\simeq}{\longrightarrow} A_{P L} \circ D^{K}
$$

of Reedy fibrant diagrams in CDGA. Their limits are therefore quasi-isomorphic, since lim is right Quillen. On the other hand, $A^{*}$ maps colimits to limits [5, §13.5]), so there exists a zig-zag of quasi-isomorphisms

$$
\lim \left(A_{P L} \circ D^{K}\right) \stackrel{\simeq}{\longrightarrow} \cdots \stackrel{\simeq}{\longleftarrow} A_{P L}\left(\mathcal{Z}_{K}\right)
$$

in CDGA, by analogy with 35, (5.6)]. Hence $\lim \left(\wedge(U) \otimes S_{K}(V)\right)$ and $A_{P L}\left(\mathcal{Z}_{K}\right)$ are weakly equivalent.

Finally, (6.6) and Proposition 4.7 provide a quasi-isomorphism

$$
\lim \left(\wedge(U) \otimes S_{K}(V)\right) \stackrel{\simeq}{\longrightarrow} \operatorname{holim} \wedge_{U / K}
$$

in CDGA.

Our two algebraic models for $\mathcal{Z}_{K}$ are consistent, in the following sense. Define $(\wedge(U) \otimes \mathbb{Q}[K], d)$ in CDGA by $d u_{j}=v_{j}$ for $1 \leq j \leq m$, and recall the quasiisomorphism $e_{K}$ of (5.6); then [16, Lemma 14.2] shows that

$$
1 \otimes e_{K}: \wedge(U) \otimes M(D J(K)) \longrightarrow \wedge(U) \otimes \mathbb{Q}[K]
$$

is a quasi-isomorphism. So $\wedge(U) \otimes \mathbb{Q}[K]$ is quasi-isomorphic to hocolim $P_{K}^{\prime}$. On the other hand, it is isomorphic to $\lim \left(\wedge(U) \otimes S_{K}(V)\right)$ because limits are created in COCH. So $\wedge(U) \otimes \mathbb{Q}[K]$ is also quasi-isomorphic to holim $\wedge_{U / K}$.

We may now retrieve the calculations of $\left[\underline{8}\right.$ for $H^{*}\left(\mathcal{Z}_{K} ; \mathbb{Q}\right)$.

Corollary 6.3. There are isomorphisms of (bi)graded algebras

$$
H^{*}\left(\mathcal{Z}_{K} ; \mathbb{Q}\right) \cong \operatorname{Tor}_{S(V)}(\mathbb{Q}[K], \mathbb{Q}) \cong H(\wedge(U) \otimes \mathbb{Q}[K], d) .
$$


Remarks 6.4. The algebra $(\wedge(U) \otimes S(\sigma), d)$ is actually the cellular cochain algebra of $\left(S^{\infty}\right)^{\sigma} \times T^{V \backslash \sigma}$, where $S^{\infty}$ has a single cell in each dimension. So the projection $\wedge(U) \otimes S(\sigma) \rightarrow \wedge\left(u_{i} \notin \sigma\right)$ is an algebraic model for the retraction $\left(S^{\infty}\right)^{\sigma} \times T^{V \backslash \sigma} \rightarrow$ $T^{V \backslash \sigma}$, given any face $\sigma$ of $K$. The corresponding model for the inclusion $D_{\sigma} \rightarrow$ $\left(S^{\infty}\right)^{\sigma} \times T^{V \backslash \sigma}$ is the projection

$$
\wedge(U) \otimes S(\sigma) \rightarrow \wedge(U) \otimes S(\sigma) /\left(u_{j} v_{j}=v_{j}^{2}=0 \text { for } j \in \sigma\right),
$$

which reflects the fact that $\left(u_{j} v_{j}=v_{j}^{2}=0\right.$ for $\left.j \in \sigma\right)$ is an acyclic ideal [3]. These models also work over $\mathbb{Z}$, and are used in [3] to establish an integral version of Corollary 6.3 (which was confirmed in [17] by other methods). The model categorical interpretation must be relaxed in this case; nevertheless, $\wedge_{R}(U) \otimes R[K]$ may still be interpreted as a homotopy limit in $\mathrm{DGA}_{R}$.

As shown in [2], the algebra $\wedge(U) \otimes \mathbb{Q}[K]$ admits non-trivial triple Massey products for certain complexes $K$, in which case $\mathcal{Z}_{K}$ cannot be formal.

We may summarise this section by combining Theorems 6.1 and 6.2

Proposition 6.5. As functors TOP $\rightarrow$ CDGA, both rational cohomology and $A_{P L}$ map homotopy limits to homotopy colimits on diagrams $P_{K}$, and map homotopy colimits to homotopy limits on diagrams $T^{V / K}$.

\section{MODELS FOR QUASITORIC MANIFOLDS}

In this section we describe the properties of Davis and Januszkiewicz's quasitoric manifolds within the model categorical framework. We prove that they are rationally formal, and extend our analysis to generalisations such as the torus manifolds of $[29$.

For any simplicial convex $n$-polytope, the Stanley-Reisner algebra of the boundary complex $K$ is Cohen-Macaulay $[7$, and $\mathbb{Q}[K]$ admits a 2-dimensional linear system of parameters $l_{1}, \ldots, l_{n}$. If the parameters are integral, then an associated quasitoric manifold $M[l]$ may be constructed; up to homotopy, it is the pullback of the diagram

$$
P_{l}:=D J(K) \stackrel{l}{\longrightarrow} B T^{n} \stackrel{p}{\longleftarrow} E T^{n},
$$

where $l$ represents the sequence $\left(l_{1}, \ldots, l_{n}\right)$ as an element of $H^{2}\left(D J(K) ; \mathbb{Z}^{n}\right)$. In these circumstances, the quotient of $M[l]$ by the canonical $T^{n}$-action is the simple polytope whose boundary is dual to $K$, and $\mathbb{Z}[K]$ is free and of finite rank over the polynomial algebra $S_{\mathbb{Z}}(L)$. Every object of TOP is fibrant and $p$ is a fibration, so there is a weak equivalence $M[l] \rightarrow \operatorname{holim} P_{l}$ by Proposition 4.8(2).

In 2-dimensional integral homology, $l$ induces $l_{*}: \mathbb{Z}^{V} \rightarrow \mathbb{Z}^{n}$, which extends to a dicharacteristic homomorphism $\ell: T^{V} \rightarrow T^{n}[9$. The kernel of $\ell$ is an $(m-n)$ dimensional subtorus $T[l]<T^{V}$, which acts freely on $\mathcal{Z}_{K}$ with quotient $M[l]$. Moreover, $\ell$ maps $T^{\sigma}$ isomorphically onto its image, which we denote by $T(\sigma)<T^{n}$ for any face $\sigma$ of $K$. We write the integral cohomology ring $H^{*}\left(T^{n} / T(\sigma)\right)$ as $\wedge(\sigma, l)$, because it is isomorphic to an exterior subalgebra on $n-|\sigma|$ generators.

Following [48, Proposition 5.3], we may also describe $M[l]$ as the homotopy colimit of the $\operatorname{CAT}(K)$-diagram $T^{n /(K, l)}$, whose value on $\sigma \subseteq \tau$ is the projection $T^{n} / T(\sigma) \rightarrow T^{n} / T(\tau)$. This result appears to be the earliest mention of homotopy colimits in the toric context, and refers to a diagram that is clearly not Reedy cofibrant. The weak equivalence of hocolim $T^{n /(K, l)}$ with the pullback of (7.1) 
follows directly from [36. Prop. 5.1], and is the quotient of the equivalence between (6.1) and (6.3) by $T[l]$, which acts freely on the entire construction.

As in Section [5, we apply $A_{P L}$ to both descriptions. We need the $\mathrm{CAT}^{o p}(K)$ diagram $\wedge_{n /(K, l)}$, which maps $\tau \supseteq \sigma$ to the inclusion $\wedge(\tau, l) \rightarrow \wedge(\sigma, l)$ in CDGA, and is clearly not Reedy fibrant. We also use the standard model [16, Chapter 15(c)] for the principal $T^{n}$-bundle of (7.1), given by the commutative diagram

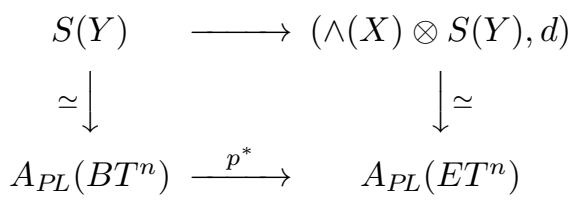

in CDGA; here $X$ and $Y$ denote sets of 1 - and 2-dimensional variables $x_{1}, \ldots, x_{n}$ and $y_{1}, \ldots, y_{n}$ respectively, with $d x_{i}=y_{i}$ for $1 \leq i \leq n$. The upper arrow is a cofibration, as described in Subsection 3.2 and $S(Y)$ is the minimal model for $B T^{n}$, as in (6.4).

We proceed by generalising arguments of Bousfield and Gugenheim [5, §16]. We work with an abitrary simply connected $\mathrm{CW}$-complex $B$ of finite type, and a set of cohomology classes $l_{1}, \ldots, l_{n}$ in $H^{2}(B ; \mathbb{Z})$. By analogy with (7.1), we let $l: B \rightarrow B T^{n}$ represent $\left(l_{1}, \ldots, l_{n}\right)$, and define $E[l]$ as the colimit of

$$
B \stackrel{l}{\longrightarrow} B T^{n} \stackrel{p}{\longleftarrow} E T^{n} .
$$

Thus $l^{*}\left(y_{i}\right)=l_{i}$ in $H^{2}(B ; \mathbb{Z})$ for $1 \leq i \leq n$, and $H^{*}(B ; \mathbb{Z})$ is an $S_{\mathbb{Z}}(Y)$-module.

Proposition 7.1. If $B$ is a formal space and $H^{*}(B)$ is free over $S(Y)$, then $E[l]$ is also formal.

Proof. The minimal model $M(B)$ reduces the zig-zag (2.4) to the form

$$
H^{*}(B) \stackrel{e}{\longleftarrow} M(B) \longrightarrow A_{P L}(B),
$$

and contains 2-dimensional cocycles $m_{i}$ such that $e\left(m_{i}\right)=l_{i}$ for $1 \leq i \leq n$. Applying $A_{P L}$ to (7.3) and importing (7.2) yields the commutative ladder

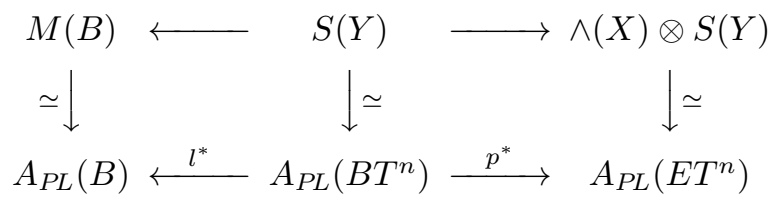

in CDGA. The colimit of the upper row is given by $(\wedge(X) \otimes M(B), d)$, where $d x_{i}=m_{i}$ for $1 \leq i \leq n$, and the colimit of the lower row maps naturally to $A_{P L}(E[l])$; so the composition $e^{\prime}: \wedge(X) \otimes M(B) \rightarrow A_{P L}(E[l])$ is defined. Since $B$ is simply connected, $e^{\prime}$ is a quasi-isomorphism by [16, $\left.\S 15(\mathrm{c})\right]$, and we obtain a zig-zag

$$
\left(\wedge(X) \otimes H^{*}(B), d\right) \stackrel{1 \otimes e}{\longleftarrow} \wedge(X) \otimes M(B) \stackrel{e^{\prime}}{\longrightarrow} A_{P L}(E[l])
$$

in CDGA, where $d x_{i}=l_{i}$ in $H^{2}(B)$ for $1 \leq i \leq n$. Furthermore, $1 \otimes e$ is a quasiisomorphism by [16, Lemma 14.2].

We now utilise the fact that $H^{*}(B)$ is free over $S(Y)$. Taking the quasi-isomorphism $\wedge(X) \otimes S(Y) \rightarrow \mathbb{Q}$ and applying the functor $\otimes_{S(Y)} H^{*}(B)$ yields a quasiisomorphism $\left(\wedge(X) \otimes H^{*}(B), d\right) \rightarrow H^{*}(B) /(Y)$, which is given by projection onto the second factor. Moreover, the Eilenberg-Moore spectral sequence confirms that the natural map $H^{*}(B) /(Y) \rightarrow H^{*}(E[l])$ is an isomorphism (as explained in 29, 
Lemma 2.1], for example). Combining the resulting quasi-isomorphism $\wedge(X) \otimes$ $H^{*}(B) \rightarrow H^{*}(E[l])$ with (7.5) then provides a zig-zag

$$
H^{*}(E[l]) \stackrel{\simeq}{\longrightarrow} \cdots \stackrel{\simeq}{\longleftarrow} A_{P L}(E[l])
$$

in $D G C A$, as required.

Corollary 7.2. Every quasitoric manifold $M[l]$ is formal, and the algebra $A_{P L}(M[l])$ is weakly equivalent to holim $\wedge_{n /(K, l)}$ in CDGA.

Proof. The formality of $A_{P L}(M[l])$ follows by applying Proposition 7.1 to (7.1), and (7.5) confirms that it is weakly equivalent to $(\wedge(X) \otimes \mathbb{Q}[K], d)$ in CDGA, where $d x_{i}=l_{i}$ for $1 \leq i \leq n$. Moreover, the natural map

$$
\wedge(X) \otimes \mathbb{Q}[K] \longrightarrow \lim \left(\wedge(X) \otimes S_{K}(Y)\right)
$$

is an isomorphism, using (5.3) and the fact that limits are created in $\mathrm{COCH}$.

Objectwise projection induces a weak equivalence $\wedge(X) \otimes S_{K}(Y) \stackrel{\simeq}{\longrightarrow} \wedge_{n /(K, l)}$ in $\left[\mathrm{CAT}^{o p}(K), \mathrm{CDGA}\right]$, whose source is Reedy fibrant; so Proposition 4.7 provides a quasi-isomorphism $\lim \left(\wedge(X) \otimes S_{K}(Y)\right) \rightarrow \operatorname{holim} \wedge_{n /(K, l)}$. The necessary zig-zag

$$
\operatorname{holim} \wedge_{n /(K, l)} \stackrel{\simeq}{\longrightarrow} \cdots \stackrel{\simeq}{\longleftarrow} A_{P L}(M[l])
$$

in CDGA is then complete.

So we have proven the following analogue of Proposition 5.1.

Proposition 7.3. As a functor TOP $\rightarrow$ CDGA, rational cohomology maps homotopy colimits to homotopy limits on diagrams $T^{n /(K, l)}$.

Proposition 7.3 also extends to the integral setting; in particular, $H^{*}(M[l] ; R)$ is isomorphic to $R[K] /(L)$ for all coefficient rings $R$.

Similar arguments apply more generally to torus manifolds over homology polytopes [29], and even to arbitrary torus manifolds with zero odd dimensional cohomology. In the latter case, $\mathbb{Z}[K]$ is replaced by the face ring $\mathbb{Z}[\mathcal{S}]$ of an appropriate simplicial poset $\mathcal{S}$. This also admits a linear system of parameters $l$ and is free over $\mathbb{Z}[L]$, so Proposition 7.1 again establishes formality. Non-singular compact toric varieties are included in this framework, although the formality of projective examples follows immediately from the fact that they are Kähler.

\section{Models For LOOP SPACES: ARBITRARY $K$}

We now turn our attention to algebraic models for the Moore loop spaces $\Omega D J(K), \Omega \mathcal{Z}_{K}$ and $\Omega M$, where $K$ is an arbitrary simplicial complex and $M$ a quasitoric manifold. Since composition of Moore loops is strictly associative, these spaces are topological monoids with identity, and are of independent interest to homotopy theorists. Their properties are considerably simplified when $K$ satisfies the requirements of a flag complex, but we postpone discussion of this situation until the following section.

Geometric models for $\Omega M$ [10] are currently under development.

Following [36, we loop the fibrations of Sections 6 and 7 to obtain fibrations

$$
\Omega \mathcal{Z}_{K} \longrightarrow \Omega D J(K) \longrightarrow T^{V} \text { and } \Omega M \longrightarrow \Omega D J(K) \stackrel{\Omega l}{\longrightarrow} T^{n} .
$$


Each of these admits a section, defined by the $m$ generators of $\pi_{2}(D J(K)) \cong \mathbb{Z}^{V}$ and by the duals of $l_{1}, \ldots, l_{n}$ respectively, and are therefore split in TOP. So we have homotopy equivalences

$$
\Omega D J(K) \stackrel{\simeq}{\longrightarrow} \Omega \mathcal{Z}_{K} \times T^{V} \text { and } \Omega D J(K) \stackrel{\simeq}{\longrightarrow} \Omega M \times T^{n}
$$

which do not preserve $H$-space structures. We therefore obtain exact sequences of Pontrjagin algebras

$$
0 \longrightarrow H_{*}(\Omega S ; R) \longrightarrow H_{*}(\Omega D J(K) ; R) \longrightarrow \wedge\left(W_{S}\right) \longrightarrow 0,
$$

where $W_{S}=U$ or $n$ as $S=\mathcal{Z}_{K}$ or $M$; these do not usually split as algebras.

Our models rely heavily on the loop and classifying functors $\Omega_{*}$ and $B_{*}$ of Subsection 3.7. For any simply-connected CW-complex $X$, the reduced singular chain complex $C_{*}(X ; R)$ is an object of DGC under the Alexander-Whitney diagonal, and Adams [1] provides a chain equivalence $\Omega_{*} C_{*}(X ; R) \rightarrow C_{*}(\Omega X ; R)$.

When $R$ is $\mathbb{Q}$, the source of the adjunction (3.3) is a full subcategory of a model category, and its target is model.

Proposition 8.1. The loop functor $\Omega_{*}$ preserves cofibrations of connected coalgebras and weak equivalences of simply connected coalgebras; the classifying functor $B_{*}$ preserves fibrations of connected algebras and all weak equivalences.

Proof. The fact that $B_{*}$ and $\Omega_{*}$ preserve weak equivalences of algebras and simply connected coalgebras respectively is proved by standard arguments with the Eilenberg-Moore spectral sequence [15, page 538]. The additional assumption for coalgebras is necessary to ensure that the cobar spectral sequence converges, because the relevant filtration is decreasing.

Given any cofibration $i: C_{1} \rightarrow C_{2}$ of connected coalgebras, we must check that $\Omega_{*} i: \Omega_{*} C_{1} \rightarrow \Omega_{*} C_{2}$ satisfies the left lifting property with respect to any acyclic fibration $p: A_{1} \rightarrow A_{2}$ in $\mathrm{DGA}_{R}$. This involves finding lifts $\Omega_{*} C_{2} \rightarrow A_{1}$ and $C_{2} \rightarrow$ $B_{*} A_{1}$ in the respective diagrams

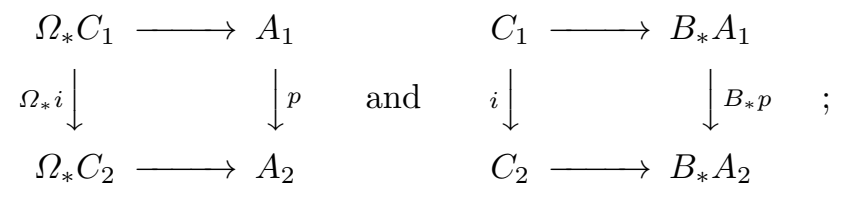

each lift implies the other, by adjointness. Since $p$ is an acyclic fibration, its kernel $A$ satisfies $H(A) \cong \mathbb{Q}$. Moreover, the projection $B_{*} p$ splits by [26, Theorem IV.2.5], so $B_{*} A_{1}$ is isomorphic to the cofree product $B_{*} A_{2} \star B_{*} A$. In this case $B_{*} p$ is an acyclic fibration in $\mathrm{DGC}_{0, R}$, and our lift is assured.

A second application of adjointness shows that $B_{*}$ preserves all fibrations of connected algebras.

Remarks 8.2. It follows from Proposition 8.1 that the restriction of (3.3) to

$$
\Omega_{*}: \mathrm{DGC}_{1, R} \rightleftarrows \mathrm{DGA}_{0, R}: B_{*}
$$

acts as a Quillen pair, and induces an adjoint pair of equivalences on appropriate full subcategories of the homotopy categories. An example is given in [15, p. 538] which shows that $\Omega_{*}$ fails to preserve quasi-isomorphisms (or even acyclic cofibrations) if the coalgebras are not simply connected. 
We now focus on the situation when $X$ is $D J(K)$. By dualising the formality results of [35, Theorem 4.8], we obtain a zig-zag of quasi-isomorphisms

$$
C_{*}(D J(K) ; \mathbb{Q}) \stackrel{\simeq}{\longrightarrow} \cdots \stackrel{\simeq}{=} Q\langle K\rangle .
$$

in DGC. Since $\Omega_{*}$ preserves quasi-isomorphisms, (8.3) combines with Adams's results [1] to determine an isomorphism

$$
H\left(\Omega_{*} \mathbb{Q}\langle K\rangle, d\right):=\operatorname{Cotor}_{\mathbb{Q}\langle K\rangle}(\mathbb{Q}, \mathbb{Q}) \stackrel{\cong}{\longrightarrow} H_{*}(\Omega D J(K) ; \mathbb{Q})
$$

of graded algebras. Our first model for $\Omega D J(K)$ is therefore $\Omega_{*} \mathbb{Q}\langle K\rangle$ in DGA.

The graded algebra underlying $\Omega_{*} R\langle K\rangle$ is the tensor algebra $T\left(s^{-1} \bar{R}\langle K\rangle\right)$ on the desuspended $R$-module $\bar{R}\langle K\rangle$; the differential is defined on generators by

$$
d\left(s^{-1} v\langle\sigma\rangle\right)=\sum_{\sigma=\tau \sqcup \tau^{\prime} ; \tau, \tau^{\prime} \neq \varnothing} s^{-1} v\langle\tau\rangle \otimes s^{-1} v\left\langle\tau^{\prime}\right\rangle,
$$

because $d=0$ on $R\langle K\rangle$. For future purposes it is convenient to write $s^{-1} v\langle\sigma\rangle$ as $\chi_{\sigma}$ for any face $\sigma \in K$, and to use the abbreviations

$$
\chi_{i}:=\chi_{v_{i}} \text { and } \chi_{i j}:=\chi_{\left\{v_{i}, v_{j}\right\}} \text { for all } 1 \leq i, j \leq m .
$$

Our aim is to construct models that satisfy algebraic analogues of [36, Theorem 7.17]. This asserts the existence of a commutative diagram

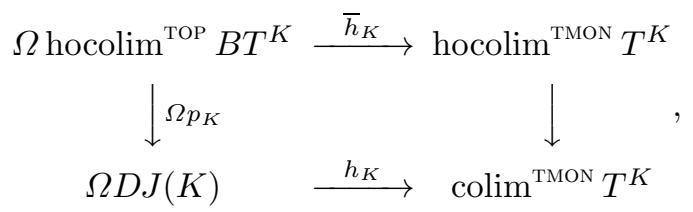

in $H o$ (TMON), where $\Omega p_{K}$ and $\bar{h}_{K}$ are homotopy equivalences for any $K$, and $h_{K}$ is a homotopy equivalence when $K$ is flag. We interpret $T^{K}$ as a diagram of topological monoids, rather than of topological spaces as in Proposition 5.1

Our previous algebraic models for $T^{K}$ have been commutative, contravariant, and cohomological, but to investigate (8.6) we introduce models that are covariant and homological. They involve the diagrams

$$
\wedge^{K}(U): \mathrm{CAT}(K) \rightarrow \mathrm{DGA} \text { and } C L^{K}(U): \mathrm{CAT}(K) \rightarrow \mathrm{DGL}
$$

which assign to $\sigma \subseteq \tau$ the monomorphisms $\wedge(\sigma) \rightarrow \wedge(\tau)$ and $C L(\sigma) \rightarrow C L(\tau)$, of exterior algebras and commutative Lie algebras respectively, on 1-dimensional generators with zero differentials. Thus $\wedge^{K}(U)$ may be identified with the diagram $H_{*}\left(T^{K} ; \mathbb{Q}\right)$ of Pontrjagin rings, and $C L^{K}(U)$ with its diagram $\pi_{*}\left(T^{K}\right) \otimes_{\mathbb{Z}} \mathbb{Q}$ of primitives. For $B T^{K}$, we consider the diagram $S^{K}\langle V\rangle: \operatorname{CAT}(K) \rightarrow$ DGC, which assigns to $\sigma \subseteq \tau$ the monomorphism $S\langle\sigma\rangle \rightarrow S\langle\tau\rangle$ of coalgebras, on 2-dimensional generators with zero differential. It may be identified with the diagram $H_{*}\left(B T^{K} ; \mathbb{Q}\right)$ of homology coalgebras.

The individual algebras and coalgebras in these diagrams are all symmetric, but the context demands they be interpreted in the non-commutative categories; this is especially important when forming limits and colimits.

Proposition 8.3. There are acyclic fibrations $\Omega_{*} S\langle V\rangle \rightarrow \wedge(U)$ in DGA and $L_{*} S\langle V\rangle \rightarrow C L(U)$ in DGL, for any set of vertices $V$. 
Proof. Using (8.5), we define the first map by $\chi_{i} \mapsto u_{i}$ for $1 \leq i \leq m$. Because

$$
d \chi_{i i}=\chi_{i} \otimes \chi_{i} \quad \text { and } \quad d \chi_{i j}=\chi_{i} \otimes \chi_{j}+\chi_{j} \otimes \chi_{i} \quad \text { for } \quad i \neq j
$$

hold in $\Omega_{*} S\langle V\rangle$, the map is consistent with the exterior relations in its target. So it is an epimorphism and quasi-isomorphism in DGA, and hence an acyclic fibration. The corresponding result for DGL follows by restriction to primitives.

By Proposition 8.3 there are acyclic fibrations $\Omega_{*} S\langle\sigma\rangle \rightarrow \wedge(\sigma)$ for every face $\sigma \in K$. These induce an acyclic Reedy fibration $e: \Omega_{*} S^{K}\langle V\rangle \rightarrow \wedge^{K}(U)$, and therefore a commutative diagram

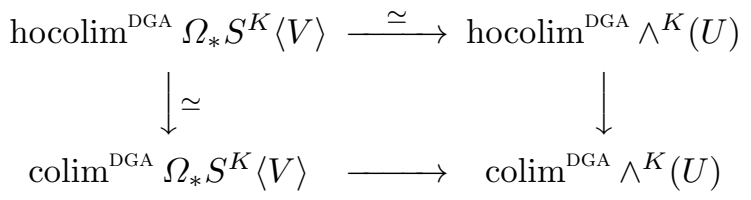

in DGA. The left-hand arrow is a quasi-isomorphism by Proposition 4.7, because $\Omega_{*} S^{K}\langle V\rangle$ is Reedy cofibrant; the upper arrow is a quasi-isomorphism by Remark 4.4, because $e$ is an equivalence.

We may now introduce our first algebraic model for diagram (8.6).

Theorem 8.4. There is a commutative diagram

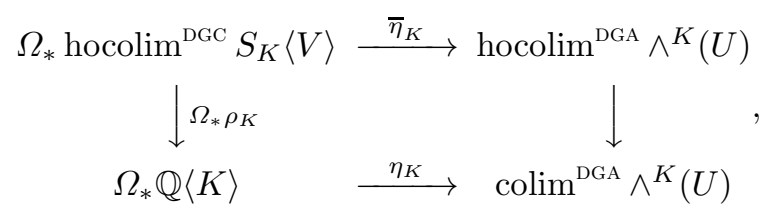

in $H o(\mathrm{DGA})$, where $\Omega_{*} \rho_{K}$ and $\bar{\eta}_{K}$ are isomorphisms for any $K$.

Proof. Consider the diagram

$$
\Omega_{*} \text { hocolim }^{\text {DGC }} S^{K}\langle V\rangle \stackrel{\simeq}{\longrightarrow} \Omega_{*} \operatorname{colim}^{\text {DGC }} S^{K}\langle V\rangle \cong \operatorname{colim}^{\text {DGA }} \Omega_{*} S^{K}\langle V\rangle
$$

in DGA. The first arrow is a quasi-isomorphism by Proposition 4.7 because $S^{K}\langle V\rangle$ is Reedy cofibrant in DGC and $\Omega_{*}$ preserves quasi-isomorphisms; the second arrow is an isomorphism because $\Omega_{*}$ is a left adjoint. So we may combine (8.9) with the upper left hand corner of (8.8) to obtain a zig-zag

$$
\Omega_{*} \text { hocolim }^{\mathrm{DGC}} S^{K}\langle V\rangle \stackrel{\simeq}{\longrightarrow} \cdots \stackrel{\simeq}{\simeq} \operatorname{hocolim}^{\mathrm{DGA}} \Omega_{*} S^{K}\langle V\rangle \stackrel{\simeq}{\longrightarrow} \operatorname{hocolim}^{\mathrm{DGA}} \wedge^{K}(U)
$$

of weak equivalences in DGA, which we label $\bar{\eta}_{K}$. The result follows by amalgamating diagrams (8.8) and (8.9), rewriting colim ${ }^{\mathrm{DGC}} S^{K}\langle V\rangle$ as $\mathbb{Q}\langle K\rangle$ throughout, and passing to the homotopy category.

By construction, $\eta_{K}$ is the fibration

$$
\Omega_{*} \mathbb{Q}\langle K\rangle \cong \Omega_{*} \operatorname{colim}^{\mathrm{DGC}} S^{K}\langle V\rangle \rightarrow \operatorname{colim}^{\mathrm{DGA}} \wedge^{K}(U)
$$

in DGA. Corollary 9.4 and Theorem 9.6 show that $\eta_{K}$ is acyclic when $K$ is flag.

The following statement is proved similarly. 
Theorem 8.5. There is a commutative diagram

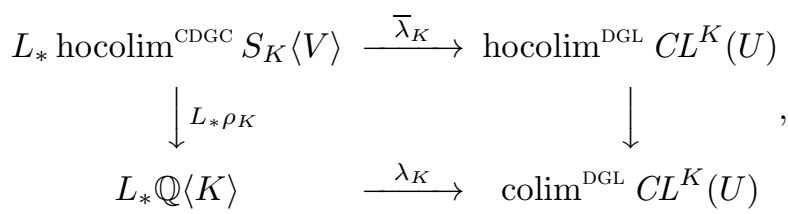

in $H o(\mathrm{DGL})$, where $L_{*} \rho_{K}$ and $\bar{\lambda}_{K}$ are isomorphisms for any $K$.

Theorem 9.6 confirms that $\lambda_{K}$ is a quasi-isomorphism when $K$ is flag.

Corollary 8.6. For any simplicial complex $K$, there are isomorphisms

$$
\begin{aligned}
H_{*}(\Omega D J(K) ; \mathbb{Q}) & \cong H\left(\text { hocolim }^{\mathrm{DGA}} \wedge^{K}(U)\right) \\
\pi_{*}(\Omega D J(K)) \otimes_{\mathbb{Z}} \mathbb{Q} & \cong H\left(\text { hocolim }^{\mathrm{DGL}} C L^{K}(U)\right)
\end{aligned}
$$

of graded algebras and Lie algebras respectively.

\section{Models for loop SpaCes: flag COMPlexes $K$}

In this section we study the loop spaces associated to flag complexes $K$. Such complexes have significantly simpler combinatorial properties, which are reflected in the homotopy theory of the toric spaces. We modify results of the previous section in this context, and focus on applications to the rational Pontrjagin rings and homotopy Lie algebras of $\Omega D J(K)$ and $\Omega \mathcal{Z}_{K}$.

For any simplicial complex $K$, a subset $\zeta \subseteq V$ is called a missing face when every proper subset lies in $K$, but $\zeta$ itself does not. If every missing face of $K$ has 2 vertices, then $K$ is a flag complex; equivalently, $K$ is flag when every set of vertices that is pairwise connected spans a simplex. A flag complex is therefore determined by its 1-skeleton, which is a graph. When $K$ is flag, we may express the Stanley-Reisner algebra as

$$
R[K]=T_{R}(V) /\left(v_{i} v_{j}-v_{j} v_{i}=0 \text { for }\{i, j\} \in K, v_{i} v_{j}=0 \text { for }\{i, j\} \notin K\right)
$$

over any ring $R$. It is therefore quadratic, in the sense that it is the quotient of a free algebra by quadratic relations.

The following result of Fröberg [18, §3] allows us to calculate the Yoneda algebras $\operatorname{Ext}_{A}(\mathbb{Q}, \mathbb{Q})$ explicitly for a class of quadratic algebras $A$ that includes StanleyReisner algebras of flag complexes.

Proposition 9.1. As graded algebras, $\operatorname{Ext}_{\mathbb{Q}[K]}(\mathbb{Q}, \mathbb{Q})$ is isomorphic to

$$
T(U) /\left(u_{i}^{2}=0, u_{i} u_{j}+u_{j} u_{i}=0 \text { for }\{i, j\} \in K\right)
$$

for any flag complex $K$.

Remark 9.2. Algebra (9.2) is the quadratic dual of (9.1). A quadratic algebra $A$ is called Koszul 43 if its quadratic dual coincides with $\operatorname{Ext}_{A}(\mathbb{Q}, \mathbb{Q})$, so Proposition 9.1 asserts that $\mathbb{Q}[K]$ is Koszul whenever $K$ is flag.

Theorem 9.3. For any flag complex $K$, there are isomorphisms

$$
\begin{aligned}
H_{*}(\Omega D J(K) ; \mathbb{Q}) & \cong T(U) /\left(u_{i}^{2}=0, u_{i} u_{j}+u_{j} u_{i}=0 \text { for }\{i, j\} \in K\right) \\
\pi_{*}(\Omega D J(K)) \otimes_{\mathbb{Z}} \mathbb{Q} & \cong F L(U) /\left(\left[u_{i}, u_{i}\right]=0,\left[u_{i}, u_{j}\right]=0 \text { for }\{i, j\} \in K\right) .
\end{aligned}
$$

Proof. The first isomorphism combines (3.4) and (8.4) with Proposition 9.1 and the second follows by restriction to primitives. 
Corollary 9.4. The algebras $H_{*}(\Omega D J(K) ; \mathbb{Q})$ and $\pi_{*}(\Omega D J(K))$ are isomorphic to colim ${ }^{\text {DGA }} \wedge^{K}(U)$ and $\operatorname{colim}^{\text {DGL }} C L^{K}(U)$ respectively, whenever $K$ is flag.

Proof. The algebras of Theorem 9.3 are the colimits of the stated diagrams, because colim $^{\text {DGA }} \wedge^{K}(U)$ is formed in the non-commutative setting of DGA.

The Poincaré series $F(\mathbb{Q}[K] ; t)$ is computed in [44, Theorem. II.1.4] for any complex $K$, although we find it convenient to adopt the re-grading associated to 1 -dimensional vertices $u_{i}$. If $K$ is $(n-1)$-dimensional and has $f_{i}$ faces of dimension $i$ for $i \geq 0$, the series becomes

$$
\sum_{i=-1}^{n-1} \frac{f_{i} t^{(i+1)}}{(1-t)^{i+1}}=\frac{h_{0}+h_{1} t+\ldots+h_{n} t^{n}}{(1-t)^{n}}
$$

where $f_{-1}=1$ and the integers $h_{i}$ are defined by (9.3) for $0 \leq i \leq n$. The integral vectors $\left(f_{-1}, \ldots, f_{n-1}\right)$ and $\left(h_{0}, \ldots, h_{n}\right)$ are known to combinatorialists as the $f$ vector and $h$-vector of $K$ respectively.

Proposition 9.5. For any flag complex $K$, we have that

$$
F\left(H_{*}(\Omega D J(K) ; \mathbb{Q}) ; t\right)=\frac{(1+t)^{n}}{1-h_{1} t+\ldots+(-1)^{n} h_{n} t^{n}} .
$$

Proof. Since $H_{*}(\Omega D J(K) ; \mathbb{Q})$ is the quadratic dual of $\mathbb{Q}[K]$, the identity

$$
F(\mathbb{Q}[K] ;-t) \cdot F\left(H_{*}(\Omega D J(K)) ; t\right)=1
$$

follows from Fröberg [18, §4]. When applied to (9.3), it yields our result.

The Poincaré series of $\pi_{*}(\Omega D J(K)) \otimes_{\mathbb{Z}} \mathbb{Q}$ is calculated in [12, §4.2].

We conclude by examining the coformality $D J(K)$, and applying the results to Theorems 8.4 and 8.5 .

Theorem 9.6. The space $D J(K)$ is coformal if and only if $K$ is flag.

Proof. If $K$ is flag, we may compose (8.10) with the first isomorphism of Corollary 9.4 to obtain an epimorphism $\Omega_{*} \mathbb{Q}\langle K\rangle \rightarrow H_{*}(\Omega D J(K) ; \mathbb{Q})$ in DGA; it is a quasi-isomorphism because both algebras have the same homology. Restricting to primitives yields a quasi-isomorphism $e: L_{*}(\mathbb{Q}\langle K\rangle) \rightarrow \pi_{*}(\Omega D J(K)) \otimes_{\mathbb{Z}} \mathbb{Q}$ in DGL, by definition of $L_{*}$.

Now choose a minimal model $M_{K} \rightarrow \mathbb{Q}[K]$ for the Stanley-Reisner algebra in CDGA. Its graded dual $\mathbb{Q}\langle K\rangle \rightarrow C_{K}$ is a minimal model for $\mathbb{Q}\langle K\rangle[34, \S 5]$ in CDGC, so $\Omega_{*} \mathbb{Q}\langle K\rangle \rightarrow \Omega_{*} C_{K}$ is a weak equivalence in DGA. Restricting to primitives provides the the central map in the zig-zag

$$
L_{K} \stackrel{\simeq}{\longrightarrow} L_{*} C_{K} \stackrel{\simeq}{\longleftarrow} L_{*} \mathbb{Q}\langle K\rangle \stackrel{e}{\longrightarrow} \pi_{*}(\Omega D J(K)) \otimes_{\mathbb{Z}} \mathbb{Q}
$$

of quasi-isomorphisms in DGL, where $L_{K}$ is a minimal model for $D J(K)$ in DGL [34, $\S 8]$. Hence $D J(K)$ is coformal.

On the other hand, every missing face of $K$ with $>2$ vertices determines a nontrivial higher Samelson bracket in $\pi_{*}(\Omega D J(K)) \otimes_{\mathbb{Z}} \mathbb{Q}$ (see Example 10.2 $(2)$ ). The existence of such brackets in $\pi_{*}(\Omega X) \otimes_{\mathbb{Z}} \mathbb{Q}$ ensures that $X$ cannot be coformal, just as higher Massey products in $H^{*}(X ; \mathbb{Q})$ obstruct formality. 


\section{EXAMPLES}

In our final section we review examples that illustrate calculations with the cobar construction, and clarify the importance of higher brackets. The examples refer to our results for flag complexes, and offer clues to the structure of Pontrjagin rings and rational homotopy Lie algebras for more general $K$.

\section{Examples 10.1.}

(1) Let $K$ be the simplex $\Delta^{n-1}$, so that $h_{0}=1$ and $h_{i}=0$ for $i>0$. Then $D J(K)$ is homeomorphic to $\left(\mathbb{C} P^{\infty}\right)^{n}$, and $\Omega D J(K)$ is homotopy equivalent to $T^{n}$; also $F\left(H_{*}(\Omega D J(K)) ; t\right)=(1+t)^{n}$, in accordance with Corollary 9.5

(2) Let $K$ be the boundary $\partial \Delta^{n}$, so that $h_{0}=\cdots=h_{n}=1$. Then $\Omega D J(K)$ is homotopy equivalent to $\Omega S^{2 n+1} \times T^{n+1}$, and

$$
F\left(H_{*}(\Omega D J(K)) ; t\right)=\frac{(1+t)^{n+1}}{1-t^{2 n}} .
$$

On the other hand, Corollary 9.5 gives

$$
\frac{(1+t)^{n}}{1-t+t^{2}+\ldots+(-1)^{n} t^{n}}=\frac{(1+t)^{n+1}}{1+(-1)^{n} t^{n+1}} \text {. }
$$

The formulae agree if $n=1$, in which case $K$ is flag, but differ otherwise.

\section{Examples 10.2.}

(1) Let $K$ be a discrete complex on $m$ vertices. Over any ring $R$, the cobar construction $\Omega_{*} R\langle K\rangle$ on the corresponding Stanley-Reisner coalgebra is generated as an algebra by the elements of the form $\chi_{i \ldots i}$ with $i \in[m]$. The first identity of (8.7) still holds, but $\chi_{i} \otimes \chi_{j}+\chi_{j} \otimes \chi_{i}$ is no longer a coboundary for $i \neq j$ since there is no element $\chi_{i j}$ in $\Omega_{*} R\langle K\rangle$. We obtain a quasi-isomorphism

$$
\Omega_{*} R\langle K\rangle \longrightarrow T_{R}\left(u_{1}, \ldots, u_{m}\right) /\left(u_{i}^{2}=0,1 \leq i \leq m\right)
$$

that maps $\chi_{i}$ to the homology class $u_{i}$. The right hand side is isomorphic to $H_{*}(\Omega D J(K) ; R)$, in accordance with Theorem 9.3 .

The space $D J(K)$ is a wedge of $m$ copies of $\mathbb{C} P^{\infty}$. In this case, $\mathcal{Z}_{K}$ is the homotopy fibre of the inclusion $\bigvee^{m} \mathbb{C} P^{\infty} \rightarrow\left(\mathbb{C} P^{\infty}\right)^{m}$ of axes, and may be identified with the complement

$$
\mathbb{C}^{m} \backslash \bigcup_{1 \leq i<j \leq m}\left\{z_{i}=z_{j}=0\right\}
$$

of the codimension-two coordinate subspaces (see [8, Example 8.15]). If $m=3$, its homotopy type may be identified by desuspending as follows:

$$
\begin{array}{r}
\mathbb{C}^{3} \backslash \bigcup_{1 \leq i<j \leq 3}\left\{z_{i}=z_{j}=0\right\} \simeq S^{5} \backslash\left(S^{1} \cup S^{1} \cup S^{1}\right) \\
\simeq \Sigma^{3}\left(S^{2} \backslash\left(S^{1} \cup S^{1} \cup S^{1}\right)\right) \\
\simeq \Sigma^{3}\left(S^{0} \vee S^{0} \vee S^{0} \vee S^{1} \vee S^{1}\right)
\end{array}
$$

where the three circles are disjoint in $S^{5}$. By [21, the complement (10.1) is homotopy equivalent to a wedge of spheres for all $m$.

The loop space $\Omega D J(K)$ is homotopy equivalent to a free product $T * \cdots * T$ of $m$ circles [36, Example 6.8], and the above calculation shows that its homology is a free product of $m$ exterior algebras on a single generator of degree 1 . Also, $\Omega \mathcal{Z}_{K}$ is homotopy equivalent to the commutator subgroup of $T * \cdots * T$. 
(2) Consider the simplest non-flag complex $K=\partial \Delta^{2}$, of Example10.1(2). Apart from the elements $\chi_{i}$ and their products in $\Omega_{*} R\left\langle\partial \Delta^{2}\right\rangle$, there is an additional 4dimensional cycle

$$
\psi:=\chi_{1} \chi_{23}+\chi_{2} \chi_{13}+\chi_{3} \chi_{12}+\chi_{12} \chi_{3}+\chi_{13} \chi_{2}+\chi_{23} \chi_{1},
$$

whose failure to bound is due to the non-existence of $\chi_{123}$. Relations (8.7) hold, and give rise to the exterior relations between the corresponding homology classes $u_{1}, u_{2}, u_{3}$. Moreover, a direct check shows that the elements $\chi_{i} \psi-\psi \chi_{i}$ are boundaries for $1 \leq i \leq 3$. We therefore have

$$
H\left(\Omega_{*} R\left\langle\partial \Delta^{2}\right\rangle\right)=H_{*}\left(\Omega D J\left(\partial \Delta^{2}\right) ; R\right) \cong \wedge\left(u_{1}, u_{2}, u_{3}\right) \otimes S(w),
$$

where $w=[\psi], \operatorname{deg} u_{i}=1, \operatorname{deg} w=4$. This calculation generalises easily to the case $K=\partial \Delta^{m-1}$ with $m \geq 3$, and we obtain similarly

$$
H_{*}\left(\Omega D J\left(\partial \Delta^{m-1}\right) ; R\right) \cong \wedge\left(u_{1}, \ldots, u_{m}\right) \otimes S(w),
$$

where $w$ is the homology class of the $(2 m-2)$-cycle $\psi \in \Omega_{*} R\left\langle\partial \Delta^{m-1}\right\rangle$, whose failure to bound is due to the non-existence of $\chi_{1 \ldots m}$. The exact sequence (8.2) takes the form

$$
0 \longrightarrow S(w) \longrightarrow H_{*}\left(\Omega D J\left(\partial \Delta^{m-1}\right) ; \mathbb{Z}\right) \longrightarrow \wedge(U) \longrightarrow 0,
$$

where $\operatorname{deg} w=2 m-2$. It follows that this sequence splits for $m \geq 3$, but not for $m=2$ as the previous calculation shows.

Unlike the situation in the previous example, there is no quasi-isomorphism $\Omega_{*} R\left\langle\partial \Delta^{m-1}\right\rangle \rightarrow H\left(\Omega_{*} R\left\langle\partial \Delta^{m-1}\right\rangle\right)$ for $m \geq 3$. The element $w$ of (10.2) is the higher commutator product; it is the Hurewicz image of the higher Samelson product of 1 -dimensional generators $u_{i} \in \pi_{1}(\Omega D J(K))$ for $1 \leq i \leq m$, see [49]. It reduces to the ordinary commutator for $m=2$. The fact that $w$ is the non-trivial higher commutator product of $u_{1}, \ldots, u_{m}$ constitutes the additional homology information necessary to distinguish between the topological monoids $\Omega D J\left(\partial \Delta^{m-1}\right)$ and $\Omega S^{2 m-1} \times T^{V}$.

(3) We finish by considering a more complicated non-flag complex, namely the 1skeleton of $\Delta^{3}$. Arguments similar to those of Example (2) show that the Pontrjagin ring $H_{*}(\Omega D J(K) ; R)$ is multiplicatively generated by four 1 -dimensional classes $u_{1}, \ldots, u_{4}$ and four 4 -dimensional classes $w_{123}, w_{124}, w_{134}, w_{234}$, corresponding to the four missing faces with three vertices each. Identities (8.7) give rise to the exterior relations between $u_{1}, \ldots, u_{4}$. We may easily check that $u_{i}$ commutes with $w_{j k l}$ if $i \in\{j, k, l\}$. The remaining non-trivial commutators are subject to one extra relation, which can be derived as follows. Consider the relation

$$
d \chi_{1234}=\left(\chi_{1} \chi_{234}+\chi_{234} \chi_{1}\right)+\ldots+\left(\chi_{4} \chi_{123}+\chi_{123} \chi_{4}\right)+\beta
$$

in $\Omega_{*} S_{R}\left\langle v_{1}, v_{2}, v_{3}, v_{4}\right\rangle$, where $\beta$ consists of terms $\chi_{\sigma} \chi_{\tau}$ such that $|\sigma|=|\tau|=2$. Denote the first four summands in the right hand side of (10.3) by $\alpha_{1}, \alpha_{2}, \alpha_{3}$, $\alpha_{4}$ respectively, and apply the differential to both sides. Observing that $d \alpha_{1}=$ $-\chi_{1} \psi_{234}+\psi_{234} \chi_{1}=-\left[\chi_{1}, \psi_{234}\right]$, and similarly for $d \alpha_{2}, d \alpha_{3}$ and $d \alpha_{4}$, we obtain

$$
\left[\chi_{1}, \psi_{234}\right]+\left[\chi_{2}, \psi_{134}\right]+\left[\chi_{3}, \psi_{124}\right]+\left[\chi_{4}, \psi_{123}\right]=d \beta .
$$

The outcome is an isomorphism

$$
H_{*}(\Omega D J(K) ; R) \cong T_{R}\left(u_{1}, u_{2}, u_{3}, u_{4}, w_{123}, w_{124}, w_{134}, w_{123}\right) / I,
$$

where $\operatorname{deg} w_{i j k}=4$ and $I$ is generated by three types of relation: 
(1) exterior algebra relations for $u_{1}, u_{2}, u_{3}, u_{4}$;

(2) $\left[u_{i}, w_{j k l}\right]=0$ for $i \in\{j, k, l\}$;

(3) $\left[u_{1}, w_{234}\right]+\left[u_{2}, w_{134}\right]+\left[u_{3}, w_{124}\right]+\left[u_{4}, w_{123}\right]=0$.

As $w_{i j k}$ is the higher commutator of $u_{i}, u_{j}$ and $u_{k},(3)$ may be considered as a higher analogue of the Jacobi identity.

\section{REFERENCES}

[1] J Frank Adams. On the cobar construction. Proc Nat Acad Sci USA, 42(7):409-412, 1956.

[2] Ilia V Baskakov. Massey triple products in the cohomology of moment-angle complexes. Russian Math Surveys 58(5): 1039-1041, 2003.

[3] Ilia V Baskakov, Victor M Buchstaber and Taras E Panov. Cellular cochain algebras and torus actions. arXiv:math.AT/0407189

[4] Hans-Joachim Baues and Jean-Michel Lemaire. Minimal models in homotopy theory. Math Ann, 225(3):219-242, 1977.

[5] A K Bousfield and Victor K Gugenheim. On PL de Rham theory and rational homotopy type. Volume 9(179) of Mem AMS, AMS, Providence, RI, 1976.

[6] A K Bousfield and Daniel M Kan. Homotopy Limits, Completions and Localizations. Volume 304 of Lecture Notes in Mathematics, Springer-Verlag, Berlin, 1972.

[7] Winfried Bruns and Hans Jørgen Herzog. Cohen-Macaulay rings. Volume 39 of Cambridge Studies in Advanced Mathematics, CUP, 1998.

[8] Victor M Buchstaber and Taras E Panov. Torus Actions and Their Applications in Topology and Combinatorics. Volume 24 of University Lecture Series, AMS, Providence RI, 2002.

[9] Victor M Buchstaber and Nigel Ray. Tangential structures on toric manifolds, and connected sums of polytopes. Internat Math Res Notices, 4:193-219, 2001.

[10] David Carter. Loops spaces on families of quasitoric manifolds. PhD Thesis, University of Manchester, 2007.

[11] Michael W Davis and Tadeusz Januszkiewicz. Convex polytopes, Coxeter orbifolds and torus actions. Duke Math J, 62:417-451, 1991.

[12] Graham Denham and Alexander I Suciu. Moment-angle complexes, monomial ideals, and Massey products. Pure and Applied Mathematics Quarterly, 3(1):25-60, 2007.

[13] William G Dwyer, Philip S Hirschhorn, Daniel M Kan and Jeffrey H Smith. Homotopy Limit Functors on Model Categories and Homotopical Categories. Volume 113 of Mathematical Surveys \& Monographs, AMS, Providence RI, 2004.

[14] William G Dwyer and J Spaliński. Homotopy Theories and Model Categories. Handbook of Algebraic Topology, Editor Ioan M James, Elsevier: 73-126, 1995.

[15] Yves Felix, Stephen Halperin and Jean-Claude Thomas. Adams' cobar equivalence. Transactions of the AMS, 329(2):531-549, 1992.

[16] Yves Félix, Stephen Halperin, and Jean-Claude Thomas. Rational Homotopy Theory, volume 205 of Graduate Texts in Mathematics. Springer Verlag, 2001.

[17] Matthias Franz. On the integral cohomology of smooth toric varieties. Volume 252 of Proc Steklov Inst Math, Moscow, 2006, pp. 53-62; math.AT/0308253

[18] Ralph Fröberg. Determination of a class of Poincaré series. Math Scand, 37:29-39, 1975.

[19] Ezra Getzler and Paul Goerss. A model category structure for differential graded coalgebras. Preprint at http://www.math.northwestern.edu/ pgoerss/, 1999.

[20] Paul G Goerss and John F Jardine. Simplicial Homotopy Theory. Volume 174 of Progress in Mathematics, Birkhäuser, 1999.

[21] Jelena Grbić and Stephen Theriault. Homotopy type of the complement of a coordinate subspace arrangement of codimension two. Russian Math Surveys, 59(6):1207-1209, 2004.

[22] Akio Hattori and Mikiya Masuda. Theory of multi-fans. Osaka J Math, 40:1-68, 2003.

[23] Philip S Hirschhorn. Model Categories and Their Localizations. Volume 99 of Mathematical Surveys and Monographs, AMS, Providence, RI, 2003.

[24] Jens Hollender and Rainer M Vogt. Modules of topological spaces, applications to homotopy colimits and $E_{\infty}$ structures. Arch Math, 59:115-129, 1992.

[25] Mark Hovey. Model Categories. Volume 63 of Mathematical Surveys and Monographs. AMS, Providence RI, 1999. 
[26] Dale Husemoller, John C Moore and James Stasheff. Differential homological algebra and homogeneous spaces. J of Pure and App Alg, 5:113-185, 1974.

[27] John F Jardine. A closed model structure for differential graded algebras. In Cyclic Cohomology and Noncommutative Geometry, volume 17 of Fields Institute Communications. AMS, 1997, pp 55-58.

[28] Saunders Mac Lane. Homology. Springer-Verlag, Berlin, 1963.

[29] Mikiya Masuda and Taras Panov. On the cohomology of torus manifolds. Osaka J Math, 43:711-746, 2006; arXiv:math.AT/0306100.

[30] J Peter May. Simplicial Objects in Algebraic Topology. Volume 11 of Van Nostrand Mathematical Studies. Van Nostrand Reinhold, 1967.

[31] J Peter May. The Geometry of Iterated Loop Spaces. Volume 11 of Lecture Notes in Math. Springer-Verlag, Berlin, 1972.

[32] John C Moore. Differential homological algebra. In Actes du Congrès International des Mathématiciens, 1970, Nice, France, volume 1. Gauthier-Villars, Paris, 1971, pp. 335-339.

[33] Joseph Neisendorfer. Lie algebras, coalgebras and rational homotopy theory for nilpotent spaces. Pac J Math, 74(2):429-460, 1978.

[34] Joseph Neisendorfer amd Timothy Miller. Formal and coformal spaces. Ill J Math, 22(4):565$580,1978$.

[35] Dietrich Notbohm and Nigel Ray. On Davis Januszkiewicz Homotopy Types. I. Formality and Rationalisation. Algebr Geom Topol, 5:31-51 (2005); arXiv:math.AT/0311167

[36] Taras Panov, Nigel Ray and Rainer Vogt. Colimits, Stanley-Reisner algebras, and loop spaces. In Categorical Decomposition Techniques in Algebraic Topology, volume 215 of Progress in Mathematics. Birkhäuser Verlag, Basel, Switzerland, 2004, pp. 261-291.

[37] Stewart Priddy. Koszul resolutions, Transactions of the AMS, 152:39-60, 1970.

[38] Daniel Quillen. Homotopical Algebra. Volume 43 of Lecture Notes in Math, Springer-Verlag, Berlin, 1967.

[39] Daniel Quillen. Rational homotopy theory, Ann Math, 2nd Ser, 90(2):205-295, 1969.

[40] Thomas Recke. Vergleich verschiedener Homotopie-Limes- und -Kolimeskonstruktionen in Modellkategorien. Diplomarbeit, Universität Osnabrück.

[41] Roland Schwanzl and Rainer M Vogt. The categories of $A_{\infty^{-}}$and $E_{\infty^{-}}$-monoids and ring spaces as closed simplicial and topological model categories, Arch Math, 56(4):405-411, 1991.

[42] Stefan Schwede and Brooke E Shipley. Algebras and modules in monoidal model categories, Proc LMS, 80:491-511, 2000.

[43] Brad Shelton and Sergey Yuzvinsky. Koszul algebras from graphs and hyperplane arrangements, J LMS, 56:477-490, 1997.

[44] Richard P Stanley. Combinatorics and Commutative Algebra, second edition. Volume 41 of Progress in Mathematics. Birkhäuser, Boston MA, 1996.

[45] Dennis Sullivan. Infinitesimal computations in topology. Institut des Hautes Études Scientifiques, Publications Mathematiques, 47:269-331, 1978.

[46] Rainer M Vogt. Convenient categories of topological spaces for homotopy theory, Arch Math, 22:545-555, 1971.

[47] Ben C Walter. Rational Homotopy Ccalculus of Functors, PhD Thesis, Brown University, Providence RI, 2005.

[48] Volkmar Welker, Günter M Ziegler and Rade T Živaljević. Homotopy colimits - comparison lemmas for combinatorial applications. Journal für die reine und angewandte Mathematik, 509:117-149, 1999.

[49] Francis D Williams. Higher Samelson products. J of Pure and App Alg, 2:249-260, 1972.

Department of Mathematics and Mechanics, Moscow State University, Leninskie Gory, 119992 Moscow, Russia;

and

Institute for Theoretical and Experimental Physics, Moscow 117259, Russia

E-mail address: tpanov@mech.math.msu.su

School of Mathematics, The University of Manchester, Oxford Road, Manchester M13 9PL, ENGLAND

E-mail address: nigel.ray@manchester.ac.uk 